\title{
UNIFORM K-STABILITY AND ASYMPTOTICS OF ENERGY FUNCTIONALS IN KÄHLER GEOMETRY WITH ERRATA
}

\author{
SÉBASTIEN BOUCKSOM, TOMOYUKI HISAMOTO, AND MATTIAS JONSSON
}

\begin{abstract}
Consider a polarized complex manifold $(X, L)$ and a ray of positive metrics on $L$ defined by a positive metric on a test configuration for $(X, L)$. For many common functionals in Kähler geometry, we prove that the slope at infinity along the ray is given by evaluating the non-Archimedean version of the functional (as defined in our earlier paper [BHJ15]) at the non-Archimedean metric on $L$ defined by the test configuration. Using this asymptotic result, we show that coercivity of the Mabuchi functional implies uniform K-stability, as defined in Der15, BHJ15.
\end{abstract}

\section{Contents}

$\begin{array}{lr}\text { About the errata } & 1 \\ \text { Introduction } & {[1} \\ \text { 1. Deligne pairings and energy functionals } \\ \text { 2. Test configurations as non-Archimedean metrics } \\ \text { 3. Non-Archimedean limits } \\ \text { 4. The logarithmic setting } \\ \text { 5. Uniform K-stability and CM-stability } \\ \text { 6. Remarks on the Yau-Tian-Donaldson conjecture } \\ \text { References }\end{array}$

AbOut THE ERRATA

An error was unfortunately found in the published version of this article, which affects a number of results of the paper. The proof of Theorem 5.6 below indeed contains an incorrect claim - but the statement of this theorem is however possibly true, and we have thus decided in this updated arXiv version to mark in red all parts of the paper affected by the issue, which are thus unproved as things stand, but hopefully repairable if the flawed proof every gets corrected. Note that we haven't tried to otherwise update the paper.

We would like to extend our warmest thanks to Yan Li, from Peking University, for pointing out this problem to us.

\section{INTRODUCTION}

Let $(X, L)$ be a polarized complex manifold, i.e. smooth projective complex variety $X$ endowed with an ample line bundle $L$. A central problem in Kähler geometry is to give

Date: May 21, 2020. 
necessary and sufficient conditions for the existence of canonical Kähler metrics in the corresponding Kähler class $c_{1}(L)$, for example, constant scalar curvature Kähler metrics (cscK for short). To fix ideas, suppose the reduced automorphism group $\operatorname{Aut}(X, L) / \mathbb{C}^{*}$ is discrete. In this case, the celebrated Yau-Tian-Donaldson conjecture asserts that $c_{1}(L)$ admits a cscK metric iff $(X, L)$ is $\mathrm{K}$-stable. That $\mathrm{K}$-stability follows from the existence of a cscK metric was proved by Stoppa [Stop09, building upon work by Donaldson [Don05], but the reverse direction is considered wide open in general.

This situation has led people to introduce stronger stability conditions that would hopefully imply the existence of a cscK metric. Building upon ideas of Donaldson [Don05], Székelyhidi Szé06] proposed to use a version of K-stability in which, for any test configuration $(\mathcal{X}, \mathcal{L})$ for $(X, L)$, the Donaldson-Futaki invariant $\operatorname{DF}(\mathcal{X}, \mathcal{L})$ is bounded below by a positive constant times a suitable norm of $(\mathcal{X}, \mathcal{L})$. (See also [Szé15] for a related notion.)

Following this lead, we defined in the prequel [BHJ15] to this paper, $(X, L)$ to be uniformly $K$-stable if there exists $\delta>0$ such that

$$
\operatorname{DF}(\mathcal{X}, \mathcal{L}) \geq \delta J^{\mathrm{NA}}(\mathcal{X}, \mathcal{L})
$$

for any normal and ample test configuration $(\mathcal{X}, \mathcal{L})$. Here $J^{\mathrm{NA}}(\mathcal{X}, \mathcal{L})$ is a non-Archimedean analogue of Aubin's $J$-functional. It is equivalent to the $L^{1}$-norm of $(\mathcal{X}, \mathcal{L})$ as well as the minimum norm considered by Dervan Der15]. The norm is zero iff the normalization of $(\mathcal{X}, \mathcal{L})$ is trivial, so uniform K-stability implies K-stability.

In [BHJ15] we advocated the point of view that a test configuration defines a nonArchimedean metric on $L$, that is, a metric on the Berkovich analytification of $(X, L)$ with respect to the trivial norm on the ground field $\mathbb{C}$. Further, we defined non-Archimedean analogues of many classical functionals in Kähler geometry. One example is the functional $J^{\mathrm{NA}}$ above. Another is $M^{\mathrm{NA}}$, a non-Archimedean analogue of the Mabuchi K-energy functional $M$. It agrees with the Donaldson-Futaki invariant, up to an explicit error term, and uniform K-stability is equivalent to

$$
M^{\mathrm{NA}}(\mathcal{X}, \mathcal{L}) \geq \delta J^{\mathrm{NA}}(\mathcal{X}, \mathcal{L})
$$

for any ample test configuration $(\mathcal{X}, \mathcal{L})$. In BHJ15] we proved that canonically polarized manifolds and polarized Calabi-Yau manifolds are always uniformly K-stable.

A first goal of this paper is to exhibit precise relations between the non-Archimedean functionals and their classical counterparts. From now on we do not a priori assume that the reduced automorphism group of $(X, L)$ is discrete. We prove

Theorem A. Let $(\mathcal{X}, \mathcal{L})$ be an ample test configuration for a polarized complex manifold $(X, L)$. Consider any smooth strictly positive $S^{1}$-invariant metric $\Phi$ on $\mathcal{L}$ defined near the central fiber, and let $\left(\phi^{s}\right)_{s}$ be the corresponding ray of smooth positive metrics on L. Denoting by $M$ and $J$ the Mabuchi K-energy functional and Aubin $J$-functional, respectively, we then have

$$
\lim _{s \rightarrow+\infty} \frac{M\left(\phi^{s}\right)}{s}=M^{\mathrm{NA}}(\mathcal{X}, \mathcal{L}) \quad \text { and } \quad \lim _{s \rightarrow+\infty} \frac{J\left(\phi^{s}\right)}{s}=J^{\mathrm{NA}}(\mathcal{X}, \mathcal{L}) .
$$

The corresponding equalities also hold for several other functionals, see Theorem 3.6. More generally, we prove that these asymptotic properties hold in the logarithmic setting, for subklt pairs $(X, B)$ and with weaker positivity assumptions, see Theorem 4.2 .

At least when the total space $\mathcal{X}$ is smooth, the assertion in Theorem A regarding the Mabuchi functional is closely related to several statements appearing in the literature [PRS08, 
Corollary 2], [PT09, Corollary 1], [Li12, Remark 12, p.38], [Tia17, Lemma 2.1], following the seminal work [Tia97]. A special case appears already in [DT92, p.328]. However, to the best of our knowledge, neither the general and precise statement given here nor its proof is available in the literature.

As in [PRS08], the proof of Theorem A uses Deligne pairings, but the analysis here is more delicate since the test configuration $\mathcal{X}$ is not smooth. Using resolution of singularities, we can make $\mathcal{X}$ smooth, but then we lose the strict positivity of $\Phi$. It turns out that the situation can be analyzed by estimating integrals of the form $\int_{\mathcal{X}_{\tau}} e^{\left.2 \Psi\right|_{\mathcal{X}_{\tau}}}$ as $\tau \rightarrow 0$, where $\mathcal{X} \rightarrow \mathbb{C}$ is an snc test configuration for $X$, and $\Psi$ is a smooth metric on the (logarithmic) relative canonical bundle of $\mathcal{X}$ near the central fiber, see Lemma 3.11.

Donaldson Don99 (see also [Mab87, Sem92]) has advocated the point of view that the space $\mathcal{H}$ of positive metrics on $L$ is an infinite-dimensional symmetric space. One can view the space $\mathcal{H}^{\mathrm{NA}}$ of positive non-Archimedean metrics on $L$ as (a subset of) the associated (conical) Tits building. Theorem A gives justification to this paradigm.

The asymptotic formulas in Theorem A allow us to study coercivity properties of the Mabuchi functional. As an immediate consequence of Theorem A, we have

Corollary B. If the Mabuchi functional is coercive in the sense that

$$
M \geq \delta J-C
$$

on $\mathcal{H}$ for some positive constants $\delta$ and $C$, then $(X, L)$ is uniformly $K$-stable, that is,

$$
\operatorname{DF}(\mathcal{X}, \mathcal{L}) \geq \delta J^{\mathrm{NA}}(\mathcal{X}, \mathcal{L})
$$

holds for any normal ample test configuration $(\mathcal{X}, \mathcal{L})$.

Coercivity of the Mabuchi functional is known to hold if $X$ is a Kähler-Einstein manifold without vector fields. This was first established in the Fano case by [PSSW08] an elegant proof can be found in DR15. As a special case of a very recent result of Berman, Darvas and $\mathrm{Lu}$ [BDL16], coercivity of the Mabuchi functional also holds for general polarized varieties admitting a metric of constant scalar curvature and having discrete reduced automorphism group. Thus, if $(X, L)$ admits a constant scalar curvature metric and $\operatorname{Aut}(X, L) / \mathbb{C}^{*}$ is discrete, then $(X, L)$ is uniformly $\mathrm{K}$-stable. The converse statement is not currently known in general, but see below for the Fano case.

Next, we study coercivity of the Mabuchi functional when restricted to the space of Bergman metrics. For any $m \geq 1$ such that $m L$ is very ample, let $\mathcal{H}_{m}$ be the space of Fubini-Study type metrics on $L$, induced by the embedding of $X \hookrightarrow \mathbb{P}^{N_{m}}$ via $m L$.

Theorem C. Fix $m$ such that $(X, m L)$ is linearly normal, and $\delta>0$. Then the following conditions are equivalent:

(i) there exists $C>0$ such that $M \geq \delta J-C$ on $\mathcal{H}_{m}$.

(ii) $\operatorname{DF}\left(\mathcal{X}_{\lambda}, \mathcal{L}_{\lambda}\right) \geq \delta J^{\mathrm{NA}}\left(\mathcal{X}_{\lambda}, \mathcal{L}_{\lambda}\right)$ for all 1-parameter subgroups $\lambda$ of $\operatorname{GL}\left(N_{m}, \mathbb{C}\right)$;

(iii) $M^{\mathrm{NA}}\left(\mathcal{X}_{\lambda}, \mathcal{L}_{\lambda}\right) \geq \delta J^{\mathrm{NA}}\left(\mathcal{X}_{\lambda}, \mathcal{L}_{\lambda}\right)$ for all 1-parameter subgroups $\lambda$ of $\mathrm{GL}\left(N_{m}, \mathbb{C}\right)$.

Here $\left(\mathcal{X}_{\lambda}, \mathcal{L}_{\lambda}\right)$ is the test configuration for $(X, L)$ defined by $\lambda$.

Note that a different condition equivalent to (i)-(iii) appears in [Pau13, Theorem 1.1].

The equivalence of (ii) and (iii) stems from the close relation between the DonaldsonFutaki invariant and the non-Archimedean Mabuchi functional. In view of Theorem A, the 
equivalence between (i) and (iii) can be viewed as a generalization of the Hilbert-Mumford criterion. The proof uses in a crucial way a deep result of Paul Pau12, which states that the restrictions to $\mathcal{H}_{m}$ of the Mabuchi functional and the $J$-functional have log norm singularities (see $\$ 5$ ).

Since every ample test configuration arises as a 1-parameter subgroup $\lambda$ of $\operatorname{GL}\left(N_{m}, \mathbb{C}\right)$ for some $m$, Theorem $\mathrm{C}$ implies

Corollary D. A polarized manifold $(X, L)$ is uniformly K-stable iff there exist $\delta>0$ and a sequence $C_{m}>0$ such that $M \geq \delta J-C_{m}$ on $\mathcal{H}_{m}$ for all sufficiently divisible $m$.

Following Paul and Tian [PT06, PT09], we say that $(X, m L)$ is CM-stable when there exist $C, \delta>0$ such that $M \geq \delta J-C$ on $\mathcal{H}_{m}$.

Corollary E. If $(X, L)$ is uniformly $K$-stable, then $(X, m L)$ is CM-stable for any sufficiently divisible positive integer $m$. Hence the reduced automorphism group is finite.

Here the last statement follows from a result by Paul [Pau13, Corollary 1.1].

Let us now comment on the relation of uniform K-stability to the existence of KählerEinstein metrics on Fano manifolds. In CDS15, Chen, Donaldson and Sun proved that a Fano manifold $X$ admits a Kähler-Einstein metric iff it is K-polystable; see also [Tia15]. Since then, several new proofs have appeared. Datar and Székelyhidi [DSz15] proved an equivariant version of the conjecture, using Aubin's original continuity method. Chen, Sun and Wang [CSW15] gave a proof using the Kähler-Ricci flow.

In BBJ15, Berman and the first and last authors of the current paper used a variational method to prove a slightly different statement: in the absence of vector fields, the existence of a Kähler-Einstein metric is equivalent to uniform K-stability. In fact, the direct implication uses Corollary B above.

In 86 we outline a different proof of the fact that a uniformly K-stable Fano manifold admits a Kähler-Einstein metric. Our method, which largely follows ideas of Tian, relies on Székelyhidi's partial $C^{0}$-estimates [Szé16] along the Aubin continuity path, together with Corollary D.

As noted above, uniform K-stability implies that the reduced automorphism group of $(X, L)$ is discrete. In the presence of vector fields, there should presumably be a natural notion of uniform K-polystability. We hope to address this in future work.

There have been several important developments since a first draft of the current paper was circulated. First, Z. Sjöström Dyrefelt [SD16] and, independently, R. Dervan and J. Ross [DR16], proved a transcendental version of Theorem A. Second, as mentioned above, it was proved in BBJ15 that in the case of a Fano manifold without holomorphic vector fields, uniform K-stability is equivalent to coercivity of the Mabuchi functional, and hence to the existence of a Kähler-Einstein metric. Finally, the results in this paper were used in [BDL16] to prove that an arbitrary polarized pair $(X, L)$ admitting a cscK metric must be K-polystable.

The organization of the paper is as follows. In the first section, we review several classical energy functionals in Kähler geometry and their interpretation as metrics on suitable Deligne pairings. Then, in \$2, we recall some non-Archimedean notions from [BHJ15]. Specifically, a non-Archimedean metric is an equivalence class of test configurations, and the non-Archimedean analogues of the energy functionals in $\$ 1$ are defined using intersection 
numbers. In $\$ 3$ we prove Theorem A relating the classical and non-Archimedean functionals via subgeodesic rays. These results are generalized to the logarithmic setting in \$4. Section5 is devoted to the relation between uniform K-stability and CM-stability. In particular, we prove Theorem C and Corollaries D and E. Finally, in $\$ 6$, we show how to use Székelyhidi's partial $C^{0}$-estimates along the Aubin continuity path together with CM-stability to prove that a uniformly K-stable Fano manifold admits a Kähler-Einstein metric.

Acknowledgment. The authors would like to thank Robert Berman for very useful discussions. The first author is also grateful to Marco Maculan, Vincent Guedj and Ahmed Zeriahi for helpful conversations. He was partially supported by the ANR projects GRACK, MACK and POSITIVE. The second author was supported by JSPS KAKENHI Grant Number 256660 and 15H06262. The last author was partially supported by NSF grant DMS-1266207, the Knut and Alice Wallenberg foundation, and the United States - Israel Binational Science Foundation.

\section{Deligne Pairings And ENERgy funCtionals}

In this section we recall the definition and main properties of the Deligne pairing, as well as its relation to classical functionals in Kähler geometry.

1.1. Metrics on line bundles. We use additive notation for line bundles and metrics. If, for $i=1,2, \phi_{i}$ is a metric on a line bundle $L_{i}$ on $X$ and $a_{i} \in \mathbb{Z}$, then $a_{1} \phi_{1}+a_{2} \phi_{2}$ is a metric on $a_{1} L_{1}+a_{2} L_{2}$. This allows us to define metrics on $\mathbb{Q}$-line bundles. A metric on the trivial line bundle will be identified with a function on $X$.

If $\sigma$ is a (holomorphic) section of a line bundle $L$ on a complex analytic space $X$, then $\log |\sigma|$ stands for the corresponding (possibly singular) metric on $L$. For any metric $\phi$ on $L$, $\log |\sigma|-\phi$ is therefore a function, and

$$
|\sigma|_{\phi}:=|\sigma| e^{-\phi}=\exp (\log |\sigma|-\phi)
$$

is the length of $\sigma$ in the metric $\phi$.

We normalize the operator $d^{c}$ so that $d d^{c}=\frac{i}{\pi} \partial \bar{\partial}$, and set (somewhat abusively)

$$
d d^{c} \phi:=-d d^{c} \log |\sigma|_{\phi}
$$

for any local trivializing section $\sigma$ of $L$. The globally defined $(1,1)$-form (or current) $d d^{c} \phi$ is the curvature of $\phi$, normalized so that it represents the (integral) first Chern class of $L$.

If $X$ is a complex manifold of dimension $n$ and $\eta$ is a holomorphic $n$-form on $X$, then

$$
|\eta|^{2}:=\frac{i^{n^{2}}}{2^{n}} \eta \wedge \bar{\eta}
$$

defines a natural (smooth, positive) volume form on $X$. More generally, there is a bijection between smooth metrics on the canonical bundle $K_{X}$ and (smooth, positive) volume forms on $X$, which associates to a smooth metric $\phi$ on $K_{X}$ the volume form $e^{2 \phi}$ locally defined by

$$
e^{2 \phi}:=|\eta|^{2} /|\eta|_{\phi}^{2}
$$

for any local section $\eta$ of $K_{X}$.

If $\omega$ is a positive $(1,1)$-form on $X$ and $n=\operatorname{dim} X$, then $\omega^{n}$ is a volume form, so $-\frac{1}{2} \log \omega^{n}$ is a metric on $-K_{X}$ in our notation. The Ricci form of $\omega$ is defined as the curvature

$$
\operatorname{Ric} \omega:=-d d^{c} \frac{1}{2} \log \omega^{n}
$$


of $\omega$ of this metric; it is thus a smooth $(1,1)$-form in the cohomology class $c_{1}(X)$ of $-K_{X}$.

If $\phi$ is a smooth positive metric on a line bundle $L$ on $X$, we denote by $S_{\phi} \in C^{\infty}(X)$ the scalar curvature of the Kähler form $d d^{c} \phi$; it satisfies

$$
S_{\phi}\left(d d^{c} \phi\right)^{n}=n \operatorname{Ric}\left(d d^{c} \phi\right) \wedge\left(d d^{c} \phi\right)^{n-1} .
$$

1.2. Deligne pairings. While the construction below works in greater generality Elk89, Zha96, MG00, we will restrict ourselves to the following setting. Let $\pi: Y \rightarrow T$ be a flat, projective morphism between smooth complex algebraic varieties, of relative dimension $n \geq 0$. Given line bundles $L_{0}, \ldots, L_{n}$ on $Y$, consider the intersection product

$$
L_{0} \cdot \ldots \cdot L_{n} \cdot[Y] \in \mathrm{CH}_{\operatorname{dim} Y-(n+1)}(Y)=\mathrm{CH}_{\operatorname{dim} T-1}(Y) .
$$

Its push-forward belongs to $\mathrm{CH}_{\operatorname{dim} T-1}(T)=\operatorname{Pic}(T)$ since $T$ is smooth, and hence defines an isomorphism class of line bundle on $T$. The Deligne pairing of $L_{0}, \ldots, L_{n}$ selects in a canonical way a specific representative of this isomorphism class, denoted by

$$
\left\langle L_{0}, \ldots, L_{n}\right\rangle_{Y / T}
$$

The pairing is functorial, multilinear, and commutes with base change. It further satisfies the following key inductive property: if $Z_{0}$ is a non-singular divisor in $Y$, flat over $T$ and defined by a section $\sigma_{0} \in H^{0}\left(Y, L_{0}\right)$, then we have a canonical identification

$$
\left\langle L_{0}, \ldots, L_{n}\right\rangle_{Y / T}=\left\langle\left. L_{1}\right|_{Z_{0}}, \ldots,\left.L_{n}\right|_{Z_{0}}\right\rangle_{Z_{0} / T}
$$

For $n=0,\left\langle L_{0}\right\rangle_{Y / T}$ coincides with the norm of $L_{0}$ with respect to the finite flat morphism $Y \rightarrow T$. These properties uniquely characterize the Deligne pairing. Indeed, writing each $L_{i}$ as a difference of very ample line bundles, multilinearity reduces the situation to the case where the $L_{i}$ are very ample. We may thus find non-singular divisors $Z_{i} \in\left|L_{i}\right|$ with $\bigcap_{i \in I} Z_{i}$ non-singular and flat over $T$ for each set $I$ of indices, and we get

$$
\left\langle L_{0}, \ldots, L_{n}\right\rangle_{Y / T}=\left\langle\left. L_{n}\right|_{Z_{0} \cap \cdots \cap Z_{n-1}}\right\rangle_{Z_{0} \cap \cdots \cap Z_{n-1} / T} .
$$

1.3. Metrics on Deligne pairings. We use [Elk90, Zha96, Mor99] as references. Given a smooth metric $\phi_{j}$ on each $L_{j}$, the Deligne pairing $\left\langle L_{0}, \ldots, L_{n}\right\rangle_{Y / T}$ can be endowed with a continuous metric

$$
\left\langle\phi_{0}, \ldots, \phi_{n}\right\rangle_{Y / T},
$$

smooth over the smooth locus of $\pi$, the construction being functorial, multilinear, and commuting with base change. It is basically constructed by requiring that

$$
\left\langle\phi_{0}, \ldots, \phi_{n}\right\rangle_{Y / T}=\left\langle\left.\phi_{1}\right|_{Z_{0}}, \ldots,\left.\phi_{n}\right|_{Z_{0}}\right\rangle_{Z_{0} / T}-\int_{Y / T} \log \left|\sigma_{0}\right|_{\phi_{0}} d d^{c} \phi_{1} \wedge \cdots \wedge d d^{c} \phi_{n}
$$

in the notation of 1.2 , with $\int_{Y / T}$ denoting fiber integration, i.e. the push-forward by $\pi$ as a current. By induction, the continuity of the metric $\left\langle\phi_{0}, \ldots, \phi_{n}\right\rangle$ reduces to that of $\int_{Y / T} \log \left|\sigma_{0}\right|_{\phi_{0}} d d^{c} \phi_{1} \wedge \cdots \wedge d d^{c} \phi_{n}$, and thus follows from [Stol66, Theorem 4.9].

Remark 1.1. As explained in [Elk90, I.1], arguing by induction, the key point in checking that (1.3) is well-defined is the following symmetry property: if $\sigma_{1} \in H^{0}\left(Y, L_{1}\right)$ is a section 
with divisor $Z_{1}$ such that both $Z_{1}$ and $Z_{0} \cap Z_{1}$ are non-singular and flat over $T$, then

$$
\begin{aligned}
& \int_{Y / T} \log \left|\sigma_{0}\right|_{\phi_{0}} d d^{c} \phi_{1} \wedge \alpha+\int_{Z_{0} / T} \log \left|\sigma_{1}\right|_{\phi_{1}} \alpha \\
= & \int_{Y / T} \log \left|\sigma_{1}\right|_{\phi_{1}} d d^{c} \phi_{0} \wedge \alpha+\int_{Z_{1} / T} \log \left|\sigma_{0}\right|_{\phi_{0}} \alpha
\end{aligned}
$$

with $\alpha=d d^{c} \phi_{2} \wedge \cdots \wedge d d^{c} \phi_{n}$. By the Lelong-Poincaré formula, the above equality reduces to

$$
\pi_{*}\left(\log \left|\sigma_{0}\right|_{\phi_{0}} d d^{c} \log \left|\sigma_{1}\right|_{\phi_{1}} \wedge \alpha\right)=\pi_{*}\left(\log \left|\sigma_{1}\right|_{\phi_{1}} d d^{c} \log \left|\sigma_{0}\right|_{\phi_{0}} \wedge \alpha\right),
$$

which holds by Stokes' formula applied to a monotone regularization of the quasi-psh functions $\log \left|\sigma_{i}\right|_{\phi_{i}}$.

Metrics on Deligne pairings satisfy the following two crucial properties, which are direct consequences of (1.3).

(i) The curvature current of $\left\langle\phi_{0}, \ldots, \phi_{n}\right\rangle_{Y / T}$ satisfies

$$
d d^{c}\left\langle\phi_{0}, \ldots, \phi_{n}\right\rangle_{Y / T}=\int_{Y / T} d d^{c} \phi_{0} \wedge \cdots \wedge d d^{c} \phi_{n},
$$

where again $\int_{Y / T}$ denotes fiber integration.

(ii) Given another smooth metric $\phi_{0}^{\prime}$ on $L_{0}$, we have the change of metric formula

$$
\left\langle\phi_{0}^{\prime}, \phi_{1}, \ldots, \phi_{n}\right\rangle_{Y / T}-\left\langle\phi_{0}, \phi_{1}, \ldots, \phi_{n}\right\rangle_{Y / T}=\int_{Y / T}\left(\phi_{0}^{\prime}-\phi_{0}\right) d d^{c} \phi_{1} \wedge \cdots \wedge d d^{c} \phi_{n} .
$$

1.4. Energy functionals. Let $(X, L)$ be a polarized manifold, i.e. a smooth projective complex variety $X$ with an ample line bundle $L$. Set

$$
V:=\left(L^{n}\right) \quad \text { and } \quad \bar{S}:=-n V^{-1}\left(K_{X} \cdot L^{n-1}\right),
$$

where $n=\operatorname{dim} X$. Denote by $\mathcal{H}$ the set of smooth positive metrics $\phi$ on $L$. For $\phi \in \mathcal{H}$, set $\mathrm{MA}(\phi):=V^{-1}\left(d d^{c} \phi\right)^{n}$. Then $\mathrm{MA}(\phi)$ is a probability measure equivalent to Lebesgue measure, and $\int_{X} S_{\phi} \mathrm{MA}(\phi)=\bar{S}$ by (1.1).

We recall the following functionals in Kähler geometry. Fix a reference metric $\phi_{\text {ref }} \in \mathcal{H}$. Our notation largely follows [BBGZ13, BBEGZ11].

(i) The Monge-Ampère energy functional is given by

$$
E(\phi)=\frac{1}{n+1} \sum_{j=0}^{n} V^{-1} \int_{X}\left(\phi-\phi_{\mathrm{ref}}\right)\left(d d^{c} \phi\right)^{j} \wedge\left(d d^{c} \phi_{\mathrm{ref}}\right)^{n-j} .
$$

(ii) The $J$-functional is a translation invariant version of $E$, defined as

$$
J(\phi):=\int_{X}\left(\phi-\phi_{\mathrm{ref}}\right) \mathrm{MA}\left(\phi_{\mathrm{ref}}\right)-E(\phi) .
$$

The closely related $I$-functional is defined by

$$
I(\phi):=\int_{X}\left(\phi-\phi_{\mathrm{ref}}\right) \mathrm{MA}\left(\phi_{\mathrm{ref}}\right)-\int_{X}\left(\phi-\phi_{\mathrm{ref}}\right) \mathrm{MA}(\phi) .
$$


(iii) For any closed $(1,1)$-form $\theta$, the $\theta$-twisted Monge-Ampère energy is given by

$$
E_{\theta}(\phi)=\frac{1}{n} \sum_{j=0}^{n-1} V^{-1} \int_{X}\left(\phi-\phi_{\mathrm{ref}}\right)\left(d d^{c} \phi\right)^{j} \wedge\left(d d^{c} \phi_{\mathrm{ref}}\right)^{n-1-j} \wedge \theta
$$

Taking $\theta:=-n \operatorname{Ric}\left(d d^{c} \phi_{\text {ref }}\right)$, we obtain the Ricci energy $R:=-E_{n \operatorname{Ric}\left(d d^{c} \phi_{\mathrm{ref}}\right)}$.

(iv) The entropy of $\phi \in \mathcal{H}$ is defined as

$$
H(\phi):=\frac{1}{2} \int_{X} \log \left[\frac{\mathrm{MA}(\phi)}{\mathrm{MA}\left(\phi_{\mathrm{ref}}\right)}\right] \mathrm{MA}(\phi)
$$

that is, (half) the relative entropy of the probability measure $\mathrm{MA}(\phi)$ with respect to $\mathrm{MA}\left(\phi_{\text {ref }}\right)$. We have $H(\phi) \geq 0$, with equality iff $\phi-\phi_{\text {ref }}$ is constant.

(v) The Mabuchi functional (or K-energy) can now be defined via the Chen-Tian formula [Che00] (see also [BB14, Proposition 3.1]) as

$$
M(\phi)=H(\phi)+R(\phi)+\bar{S} E(\phi) .
$$

These functionals vanish at $\phi_{\text {ref }}$ and satisfy the variational formulas:

$$
\begin{aligned}
\delta E(\phi) & =\operatorname{MA}(\phi)=V^{-1}\left(d d^{c} \phi\right)^{n} \\
\delta E_{\theta}(\phi) & =V^{-1}\left(d d^{c} \phi\right)^{n-1} \wedge \theta \\
\delta R(\phi) & =-n V^{-1}\left(d d^{c} \phi\right)^{n-1} \wedge \operatorname{Ric}\left(d d^{c} \phi_{\mathrm{ref}}\right) \\
\delta H(\phi) & =n V^{-1}\left(d d^{c} \phi\right)^{n-1} \wedge\left(\operatorname{Ric}\left(d d^{c} \phi_{\mathrm{ref}}\right)-\operatorname{Ric}\left(d d^{c} \phi\right)\right) \\
\delta M(\phi) & =\left(\bar{S}-S_{\phi}\right) \operatorname{MA}(\phi)
\end{aligned}
$$

In particular, $\phi$ is a critical point of $M$ iff $d d^{c} \phi$ is a $\operatorname{cscK}$ metric.

The functionals $I, J$ and $I-J$ are comparable in the sense that

$$
\frac{1}{n} J \leq I-J \leq n J
$$

on $\mathcal{H}$. For $\phi \in \mathcal{H}$ we have $J(\phi) \geq 0$, with equality iff $\phi-\phi_{\text {ref }}$ is constant. These properties are thus also shared by $I$ and $I-J$.

The functionals $H, I, J, M$ are translation invariant in the sense that $H(\phi+c)=H(\phi)$ for $c \in \mathbb{R}$. For $E$ and $R$ we instead have $E(\phi+c)=E(\phi)+c$ and $R(\phi+c)=R(\phi)-\bar{S} c$, respectively.

1.5. Energy functionals as Deligne pairings. The functionals above can be expressed using Deligne pairings, an observation going back at least to [PS04]. Note that any metric $\phi \in \mathcal{H}$ induces a smooth metric $\frac{1}{2} \log \mathrm{MA}(\phi)$ on $K_{X}$. The following identities are now easy consequences of the change of metric formula 1.5 . 
Lemma 1.2. For any $\phi \in \mathcal{H}$ we have

$$
\begin{aligned}
(n+1) V E(\phi) & =\left\langle\phi^{n+1}\right\rangle_{X}-\left\langle\phi_{\mathrm{ref}}^{n+1}\right\rangle_{X} ; \\
V J(\phi) & =\left\langle\phi, \phi_{\mathrm{ref}}^{n}\right\rangle_{X}-\left\langle\phi_{\mathrm{ref}}^{n+1}\right\rangle_{X}-\frac{1}{n+1}\left[\left\langle\phi^{n+1}\right\rangle_{X}-\left\langle\phi_{\mathrm{ref}}^{n+1}\right\rangle_{X}\right] ; \\
V I(\phi) & =\left\langle\phi-\phi_{\mathrm{ref}}, \phi_{\mathrm{ref}}^{n}\right\rangle_{X}-\left\langle\phi-\phi_{\mathrm{ref}}, \phi^{n}\right\rangle_{X} ; \\
V R(\phi) & =\left\langle\frac{1}{2} \log \mathrm{MA}\left(\phi_{\mathrm{ref}}\right), \phi^{n}\right\rangle_{X}-\left\langle\frac{1}{2} \log \mathrm{MA}\left(\phi_{\mathrm{ref}}\right), \phi_{\mathrm{ref}}^{n}\right\rangle_{X} ; \\
V H(\phi) & =\left\langle\frac{1}{2} \log \mathrm{MA}(\phi), \phi^{n}\right\rangle_{X}-\left\langle\frac{1}{2} \log \mathrm{MA}\left(\phi_{\mathrm{ref}}\right), \phi^{n}\right\rangle_{X} ; \\
V M(\phi) & =\left\langle\frac{1}{2} \log \mathrm{MA}(\phi), \phi^{n}\right\rangle_{X}-\left\langle\frac{1}{2} \log \mathrm{MA}\left(\phi_{\mathrm{ref}}\right), \phi_{\mathrm{ref}}^{n}\right\rangle_{X} \\
& +\frac{\bar{S}}{n+1}\left[\left\langle\phi^{n+1}\right\rangle_{X}-\left\langle\phi_{\mathrm{ref}}^{n+1}\right\rangle_{X}\right],
\end{aligned}
$$

where \langle\rangle$_{X}$ denotes the Deligne pairing with respect to the constant map $X \rightarrow\{\mathrm{pt}\}$.

Remark 1.3. The formulas above make it evident that instead of fixing a reference metric $\phi_{\text {ref }} \in \mathcal{H}$, we could view $E, H+R$ and $M$ as metrics on suitable multiples of the complex lines $\left\langle L^{n+1}\right\rangle_{X},\left\langle K_{X}, L^{n}\right\rangle_{X}$, and $(n+1)\left\langle K_{X}, L^{n}\right\rangle_{X}+\bar{S}\left\langle L^{n+1}\right\rangle_{X}$, respectively.

Remark 1.4. In the definition of $R$, we could replace - Ric $d d^{c} \phi_{\text {ref }}$ by $d d^{c} \psi_{\text {ref }}$ for any smooth metric $\psi_{\text {ref }}$ on $K_{X}$. Similarly, in the definition of $H$, we could replace the reference measure $\mathrm{MA}\left(\phi_{\mathrm{ref}}\right)$ by $e^{2 \psi_{\mathrm{ref}}}$. Doing so, and keeping the Chen-Tian formula, would only change the Mabuchi functional $M$ by an additive constant.

1.6. The Ding functional. Now suppose $X$ is a Fano manifold, that is, $L:=-K_{X}$ is ample. Any metric $\phi$ on $L$ then induces a positive volume form $e^{-2 \phi}$ on $X$. The Ding functional Din88] on $\mathcal{H}$ is defined by

$$
D(\phi)=L(\phi)-E(\phi),
$$

where

$$
L(\phi)=-\frac{1}{2} \log \int_{X} e^{-2 \phi} .
$$

This functional has proven an extremely useful tool for the study of the existence of KählerEinstein metrics, which are realized as the critical points of $D$, see e.g. Berm16, BBJ15.

\section{Test CONFIGURATiOns AS NON-ARCHIMEDEAN METRICS}

In this section we recall some notions and results from [BHJ15]. Let $X$ be a smooth projective complex variety and $L$ a line bundle on $X$.

2.1. Test configurations. As in [BHJ15] we adopt the following flexible terminology for test configurations.

Definition 2.1. A test configuration $\mathcal{X}$ for $X$ consists of the following data:

(i) a flat, projective morphism of schemes $\pi: \mathcal{X} \rightarrow \mathbb{C}$;

(ii) a $\mathbb{C}^{*}$-action on $\mathcal{X}$ lifting the canonical action on $\mathbb{C}$;

(iii) an isomorphism $\mathcal{X}_{1} \simeq X$. 
We denote by $\tau$ the coordinate on $\mathbb{C}$, and by $\mathcal{X}_{\tau}$ the fiber over $\tau$.

These conditions imply that $\mathcal{X}$ is reduced and irreducible [BHJ15, Proposition 2.6]). If $\mathcal{X}, \mathcal{X}^{\prime}$ are test configurations for $X$, then there is a unique $\mathbb{C}^{*}$-equivariant birational map $\mathcal{X}^{\prime} \rightarrow \mathcal{X}$ compatible with the isomorphism in (iii). We say that $\mathcal{X}^{\prime}$ dominates $\mathcal{X}$ if this birational map is a morphism; when it is an isomorphism we somewhat abusively identify $\mathcal{X}$ and $\mathcal{X}^{\prime}$. Any test configuration $\mathcal{X}$ is dominated by its normalization $\widetilde{\mathcal{X}}$.

An snc test configuration for $X$ is a smooth test configuration $\mathcal{X}$ whose central fiber $\mathcal{X}_{0}$ has simple normal crossing support (but is not necessarily reduced).

When $\mathcal{X}$ is a test configuration, we define the logarithmic canonical bundle as

$$
K_{\mathcal{X}}^{\log }:=K_{\mathcal{X}}+\mathcal{X}_{0, \text { red }}
$$

Setting $K_{\mathbb{C}}^{\log }:=K_{\mathbb{C}}+[0]$, we define the relative logarithmic canonical bundle as

$$
K_{\mathcal{X} / \mathbb{C}}^{\log }:=K_{\mathcal{X}}^{\log }-\pi^{*} K_{\mathbb{C}}^{\log }=K_{\mathcal{X} / \mathbb{C}}+\mathcal{X}_{0, \text { red }}-\mathcal{X}_{0}
$$

this is well behaved under base change $\tau \mapsto \tau^{d}$, see [BHJ15, §4.4]. Despite the terminology, $K_{\mathcal{X}}, K_{\mathcal{X} / \mathbb{C}}, K_{\mathcal{X}}^{\log }$ and $K_{\mathcal{X} / \mathbb{C}}^{\log }$ are only Weil divisor classes in general; they are line bundles when $\mathcal{X}$ is smooth.

Definition 2.2. A test configuration $(\mathcal{X}, \mathcal{L})$ for $(X, L)$ consists of a test configuration $\mathcal{X}$ for $X$, together with the following additional data:

(iv) a $\mathbb{C}^{*}$-linearized $\mathbb{Q}$-line bundle $\mathcal{L}$ on $\mathcal{X}$;

(v) an isomorphism $\left(\mathcal{X}_{1}, \mathcal{L}_{1}\right) \simeq(X, L)$.

A pull-back of a test configuration $(\mathcal{X}, \mathcal{L})$ is a test configuration $\left(\mathcal{X}^{\prime}, \mathcal{L}^{\prime}\right)$ where $\mathcal{X}^{\prime}$ dominates $\mathcal{X}$ and $\mathcal{L}^{\prime}$ is the pull-back of $\mathcal{L}$. In particular, the normalization $(\tilde{\mathcal{X}}, \tilde{\mathcal{L}})$ is the pull-back of $(\mathcal{X}, \mathcal{L})$ with $\nu: \widetilde{\mathcal{X}} \rightarrow \mathcal{X}$ the normalization morphism.

A test configuration $(\mathcal{X}, \mathcal{L})$ is trivial if $\mathcal{X}=X \times \mathbb{C}$ with $\mathbb{C}^{*}$ acting trivially on $X$. This implies that $\left(\mathcal{X}, \mathcal{L}+c \mathcal{X}_{0}\right)=(X, L) \times \mathbb{C}$ for some constant $c \in \mathbb{Q}$. A test configuration for $(X, L)$ is almost trivial if its normalization is trivial.

We say that $(\mathcal{X}, \mathcal{L})$ is ample (resp. semiample, resp. nef) when $\mathcal{L}$ is relatively ample (resp. relatively semiample, resp. nef). The pullback of a semiample (resp. nef) test configuration is semiample (resp. nef).

If $L$ is ample, then for every semiample test configuration $(\mathcal{X}, \mathcal{L})$ there exists a unique ample test configuration $\left(\mathcal{X}_{\text {amp }}, \mathcal{L}_{\text {amp }}\right)$ that is dominated by $(\mathcal{X}, \mathcal{L})$ and satisfies $\mu_{*} \mathcal{O}_{\mathcal{X}}=$ $\mathcal{O}_{\mathcal{X}_{\text {amp }}}$, where $\mu: \mathcal{X} \rightarrow \mathcal{X}_{\text {amp }}$ is the canonical morphism; see [BHJ15, Proposition 2.17].

Note that, while $\mathcal{X}$ can often be chosen smooth, $\mathcal{X}_{\text {amp }}$ will not be smooth, in general. It is, however, normal whenever $\mathcal{X}$ is.

2.2. One-parameter subgroups. Suppose $L$ is ample. Ample test configurations are then essentially equivalent to one-parameter degenerations of $X$. See [BHJ15, $\S 2.3$ ] for details on what follows.

Fix $m \geq 1$ such that $m L$ is very ample, and consider the corresponding closed embedding $X \hookrightarrow \mathbb{P}^{N_{m}-1}$ with $N_{m}:=h^{0}(X, m L)$. Then every 1-parameter subgroup (1-PS for short) $\lambda: \mathbb{C}^{*} \rightarrow \operatorname{GL}\left(N_{m}, \mathbb{C}\right)$ induces an ample test configuration $\left(\mathcal{X}_{\lambda}, \mathcal{L}_{\lambda}\right)$ for $(X, L)$. By definition, $\mathcal{X}_{\lambda}$ is the Zariski closure in $\mathbb{P} V \times \mathbb{C}$ of the image of the closed embedding $X \times \mathbb{C}^{*} \hookrightarrow \mathbb{P} V \times \mathbb{C}^{*}$ mapping $(x, \tau)$ to $(\lambda(\tau) x, \tau)$. Note that $\left(\mathcal{X}_{\lambda}, \mathcal{L}_{\lambda}\right)$ is trivial iff $\lambda$ is a multiple of the identity. We emphasize that $\mathcal{X}_{\lambda}$ is not normal in general. 
In fact, every ample test configuration may be obtained as above. Using one-parameter subgroups, we can produce test configurations that are almost trivial but not trivial, as observed in [LX14, Remark 5]. See [BHJ15, Proposition 2.12] for an elementary proof of the following result.

Proposition 2.3. For every $m$ divisible enough, there exists a $1-P S \lambda: \mathbb{C}^{*} \rightarrow \operatorname{GL}\left(N_{m}, \mathbb{C}\right)$ such that the test configuration $\left(\mathcal{X}_{\lambda}, \mathcal{L}_{\lambda}\right)$ is nontrivial but almost trivial.

2.3. Valuations and log discrepancies. By a valuation on $X$ we mean a real-valued valuation $v$ on the function field $\mathbb{C}(X)$ (trivial on the ground field $\mathbb{C}$ ). The trivial valuation $v_{\text {triv }}$ is defined by $v_{\text {triv }}(f)=0$ for $f \in \mathbb{C}(X)^{*}$. A valuation $v$ is divisorial if it is of the form $v=c \operatorname{ord}_{F}$, where $c \in \mathbb{Q}_{>0}$ and $F$ is a prime divisor on a projective normal variety $Y$ admitting a birational morphism onto $X$. We denote by $X^{\text {div }}$ the set of valuations on $X$ that are either divisorial or trivial, and equip it with the weakest topology such that $v \mapsto v(f)$ is continuous for every $f \in \mathbb{C}(X)^{*}$.

The $\log$ discrepancy $A_{X}(v)$ of a valuation in $X^{\text {div }}$ is defined as follows. First, $A_{X}\left(v_{\text {triv }}\right)=$ 0 . For $v=c \operatorname{ord}_{F}$ a divisorial valuation as above, we set $A_{X}=c\left(1+\operatorname{ord}_{F}\left(K_{Y / X}\right)\right)$, where $K_{Y / X}$ is the relative canonical (Weil) divisor.

Now consider a normal test configuration $\mathcal{X}$ of $X$. Since $\mathbb{C}(\mathcal{X}) \simeq \mathbb{C}(X)(\tau)$, any valuation $w$ on $\mathcal{X}$ restricts to a valuation $r(w)$ on $X$. Let $E$ be an irreducible component of the central fiber $\mathcal{X}_{0}=\sum b_{E} E$. Then $\operatorname{ord}_{E}$ is a $\mathbb{C}^{*}$-invariant divisorial valuation on $\mathbb{C}(\mathcal{X})$ and satisfies $\operatorname{ord}_{E}(t)=b_{E}$. If we set $v_{E}:=r\left(b_{E}^{-1} \operatorname{ord}_{E}\right)$, then $v_{E}$ is a valuation in $X^{\text {div }}$. Conversely, every valuation $v \in X^{\text {div }}$ has a unique $\mathbb{C}^{*}$-invariant preimage $w$ under $r$ normalized by $w(\tau)=1$, and $w$ is associated to an irreducible component of the central fiber of some test configuration for $X$, cf. [BHJ15, Theorem 4.6].

Note that $\operatorname{ord}_{E}$ is a divisorial valuation on $X \times \mathbb{C}$. By [BHJ15, Proposition 4.11], the log discrepancies of $\operatorname{ord}_{E}$ and $v_{E}$ are related as follows: $A_{X \times \mathbb{C}}\left(\operatorname{ord}_{E}\right)=b_{E}\left(1+A_{X}\left(v_{E}\right)\right)$.

2.4. Compactifications. For some purposes it is convenient to compactify test configurations. The following notion provides a canonical way of doing so.

Definition 2.4. The compactification $\overline{\mathcal{X}}$ of a test configuration $\mathcal{X}$ for $X$ is defined by gluing together $\mathcal{X}$ and $X \times\left(\mathbb{P}^{1} \backslash\{0\}\right)$ along their respective open subsets $\mathcal{X} \backslash \mathcal{X}_{0}$ and $X \times(\mathbb{C} \backslash\{0\})$, using the canonical $\mathbb{C}^{*}$-equivariant isomorphism $\mathcal{X} \backslash \mathcal{X}_{0} \simeq X \times(\mathbb{C} \backslash\{0\})$.

The compactification $\overline{\mathcal{X}}$ comes with a $\mathbb{C}^{*}$-equivariant flat morphism $\overline{\mathcal{X}} \rightarrow \mathbb{P}^{1}$, still denoted by $\pi$. By construction, $\pi^{-1}\left(\mathbb{P}^{1} \backslash\{0\}\right)$ is $\mathbb{C}^{*}$-equivariantly isomorphic to $X \times\left(\mathbb{P}^{1} \backslash\{0\}\right)$ over $\mathbb{P}^{1} \backslash\{0\}$.

Similarly, a test configuration $(\mathcal{X}, \mathcal{L})$ for $(X, L)$ admits a compactification $(\overline{\mathcal{X}}, \overline{\mathcal{L}})$, where $\overline{\mathcal{L}}$ is a $\mathbb{C}^{*}$-linearized $\mathbb{Q}$-line bundle on $\overline{\mathcal{X}}$. Note that $\overline{\mathcal{L}}$ is relatively (semi)ample iff $\mathcal{L}$ is.

The relative canonical differential and relative canonical differential are now defined by

$$
\begin{gathered}
K_{\overline{\mathcal{X}} / \mathbb{P}^{1}}:=K_{\overline{\mathcal{X}}}-\pi^{*} K_{\mathbb{P}^{1}} \\
K_{\overline{\mathcal{X}} / \mathbb{P}^{1}}^{\log }:=K_{\overline{\mathcal{X}}}^{\log }-\pi^{*} K_{\mathbb{P}^{1}}^{\log }=K_{\overline{\mathcal{X}} / \mathbb{P}^{1}}+\mathcal{X}_{0, \text { red }}-\mathcal{X}_{0} .
\end{gathered}
$$

2.5. Non-Archimedean metrics. Following [BHJ15, §6] (see also [BJ16b]) we introduce:

Definition 2.5. Two test configurations $\left(\mathcal{X}_{1}, \mathcal{L}_{1}\right),\left(\mathcal{X}_{2}, \mathcal{L}_{2}\right)$ for $(X, L)$ are equivalent if there exists a test configuration $\left(\mathcal{X}_{3}, \mathcal{L}_{3}\right)$ that is a pull-back of both $\left(\mathcal{X}_{1}, \mathcal{L}_{1}\right)$ and $\left(\mathcal{X}_{2}, \mathcal{L}_{2}\right)$. An 
equivalence class is called a non-Archimedean metric on $L$, and is denoted by $\phi$. We denote by $\phi_{\text {triv }}$ the equivalence class of the trivial test configuration $(X, L) \times \mathbb{C}$.

A non-Archimedean metric $\phi$ is called semipositive if some (or, equivalently, any) representative $(\mathcal{X}, \mathcal{L})$ of $\phi$ is nef. Note that this implies that $L$ is nef.

When $L$ is ample, we say that a non-Archimedean metric $\phi$ on $L$ is positive if some (or, equivalently, any) representative $(\mathcal{X}, \mathcal{L})$ of $\phi$ is semiample. We denote by $\mathcal{H}^{\mathrm{NA}}$ the set of all non-Archimedean positive metrics on $L$. By [BHJ15, Lemma 6.3], every $\phi \in \mathcal{H}^{\mathrm{NA}}$ is represented by a unique normal, ample test configuration.

The set of non-Archimedean metrics on a line bundle $L$ admits two natural operations:

(i) a translation action of $\mathbb{Q}$, denoted by $\phi \mapsto \phi+c$, and induced by $(\mathcal{X}, \mathcal{L}) \mapsto(\mathcal{X}, \mathcal{L}+$ $\left.c \mathcal{X}_{0}\right)$

(ii) a scaling action of the semigroup $\mathbb{N}^{*}$ of positive integers, denoted by $\phi \mapsto \phi_{d}$ and induced by the base change of $(\mathcal{X}, \mathcal{L})$ by $\tau \mapsto \tau^{d}$.

When $L$ is ample (resp. nef) these operations preserve the set of positive (resp. semipositive) metrics. The trivial metric $\phi_{\text {triv }}$ is fixed by the scaling action.

As in 1.1 we use additive notation for non-Archimedean metrics. A non-Archimedean metric on $\mathcal{O}_{X}$ induces a bounded (and continuous) function on $X^{\text {div }}$.

Remark 2.6. As explained in [BHJ15, §6.8], a non-Archimedean metric $\phi$ on L, as defined above, can be viewed as a metric on the Berkovich analytification [Berk90] of $L$ with respect to the trivial absolute value on the ground field $\mathbb{C}$. See also BJ16b] for a more systematic analysis, itself building upon [BFJ16, BFJ15a].

2.6. Intersection numbers and Monge-Ampère measures. Following [BHJ15, §6.6] we define the intersection number $\left(\phi_{0} \cdot \ldots \cdot \phi_{n}\right)$ of non-Archimedean metrics $\phi_{0}, \ldots, \phi_{n}$ on line bundles $L_{0}, \ldots, L_{n}$ on $X$ as follows. Pick representatives $\left(\mathcal{X}, \mathcal{L}_{i}\right)$ of $\phi_{i}, 0 \leq i \leq n$, with the same test configuration $\mathcal{X}$ for $X$ and set

$$
\left(\phi_{0} \cdot \ldots \cdot \phi_{n}\right):=\left(\overline{\mathcal{L}}_{0} \cdot \ldots \cdot \overline{\mathcal{L}}_{n}\right)
$$

where $\left(\overline{\mathcal{X}}, \overline{\mathcal{L}}_{i}\right)$ is the compactification of $\left(\mathcal{X}, \mathcal{L}_{i}\right)$. It follows from the projection formula that this does not depend of the choice of the $\mathcal{L}_{i}$. Note that $\left(\phi_{\text {triv }}^{n+1}\right)=0$. When $L_{0}=\mathcal{O}_{X}$, so that $\mathcal{L}_{0}=\mathcal{O}_{X}(D)$ for a $\mathbb{Q}$-Cartier $\mathbb{Q}$-divisor $D=\sum r_{E} E$ supported on $\mathcal{X}_{0}$, we can compute the intersection number as $\left(\phi_{0} \cdot \ldots \cdot \phi_{n}\right)=\sum_{E} r_{E}\left(\left.\left.\mathcal{L}_{1}\right|_{E} \cdot \ldots \cdot \mathcal{L}_{n}\right|_{E}\right)$.

To a non-Archimedean metric $\phi$ on a big and nef line bundle $L$ on $X$ we associate, as in [BHJ15, §6.7], a signed finite atomic Monge-Ampère measure on $X^{\text {div }}$. Pick a representative $\left(\mathcal{X}, \mathcal{L}_{i}\right)$ of $\phi$, and set

$$
\mathrm{MA}^{\mathrm{NA}}(\phi)=V^{-1} \sum_{E} b_{E}\left(\left.\mathcal{L}\right|_{E} ^{n}\right) \delta_{v_{E}}
$$

where $E$ ranges over irreducible components of $\mathcal{X}_{0}=\sum_{E} b_{E} E, v_{E}=r\left(b_{E}^{-1} \operatorname{ord}_{E}\right) \in X^{\text {div }}$, and $V=\left(L^{n}\right)$. When the $\phi_{i}$ are semipositive, the mixed Monge-Ampère measure is a probability measure.

2.7. Functionals on non-Archimedean metrics. Following [BHJ15, §7] we define nonArchimedean analogues of the functionals considered in $\$ 1.4$. Fix a line bundle $L$. 
Definition 2.7. Let $W$ be a set of non-Archimedean metrics on $L$ that is closed under translation and scaling. A functional $F: W \rightarrow \mathbb{R}$ is

(i) homogeneous if $F\left(\phi_{d}\right)=d F(\phi)$ for $\phi \in W$ and $d \in \mathbb{N}^{*}$;

(ii) translation invariant if $F(\phi+c)=F(\phi)$ for $\phi \in W$ and $c \in \mathbb{Q}$.

When $L$ is ample, a functional $F$ on $\mathcal{H}^{\mathrm{NA}}$ may be viewed as a function $F(\mathcal{X}, \mathcal{L})$ on the set of all semiample test configurations $(\mathcal{X}, \mathcal{L})$ that is invariant under pull-back, i.e. $F\left(\mathcal{X}^{\prime}, \mathcal{L}^{\prime}\right)=F(\mathcal{X}, \mathcal{L})$ whenever $\left(\mathcal{X}^{\prime}, \mathcal{L}^{\prime}\right)$ is a pull-back of a $(\mathcal{X}, \mathcal{L})($ and, in particular, invariant under normalization). Homogeneity amounts to $F\left(\mathcal{X}_{d}, \mathcal{L}_{d}\right)=d F(\mathcal{X}, \mathcal{L})$ for all $d \in \mathbb{N}^{*}$, and translation invariance to $F(\mathcal{X}, \mathcal{L})=F(\mathcal{X}, \mathcal{L}+c \mathcal{X} 0)$ for all $c \in \mathbb{Q}$.

For each non-Archimedean metric $\phi$ on $L$, choose a normal representative $(\mathcal{X}, \mathcal{L})$ that dominates $X \times \mathbb{C}$ via $\rho: \mathcal{X} \rightarrow X \times \mathbb{C}$. Then $\mathcal{L}=\rho^{*}(L \times \mathbb{C})+D$ for a uniquely determined $\mathbb{Q}$-Cartier divisor $D$ supported on $\mathcal{X}_{0}$. Write $\mathcal{X}_{0}=\sum_{E} b_{E} E$ and let $(\overline{\mathcal{X}}, \overline{\mathcal{L}})$ be the compactification of $(\mathcal{X}, \mathcal{L})$.

In this notation, we may describe our list of non-Archimedean functionals as follows. Assume $L$ is big and nef. Let $\phi_{\text {triv }}$ and $\psi_{\text {triv }}$ be the trivial metrics on $L$ and $K_{X}$, respectively.

(i) The non-Archimedean Monge-Ampère energy of $\phi$ is

$$
\begin{aligned}
E^{\mathrm{NA}}(\phi): & =\frac{\left(\phi^{n+1}\right)}{(n+1) V} \\
& =\frac{\left(\overline{\mathcal{L}}^{n+1}\right)}{(n+1) V} .
\end{aligned}
$$

(ii) The non-Archimedean I-functional and $J$-functional are given by

$$
\begin{aligned}
I^{\mathrm{NA}}(\phi): & =V^{-1}\left(\phi \cdot \phi_{\text {triv }}^{n}\right)-V^{-1}\left(\left(\phi-\phi_{\text {triv }}\right) \cdot \phi^{n}\right) \\
& =V^{-1}\left(\overline{\mathcal{L}} \cdot\left(\rho^{*}\left(L \times \mathbb{P}^{1}\right)^{n}\right)-V^{-1}\left(D \cdot \overline{\mathcal{L}}^{n}\right) .\right.
\end{aligned}
$$

and

$$
\begin{aligned}
J^{\mathrm{NA}}(\phi): & =V^{-1}\left(\phi \cdot \phi_{\text {triv }}^{n}\right)-E^{\mathrm{NA}}(\phi) \\
& =\frac{1}{V}\left(\overline{\mathcal{L}} \cdot\left(\rho^{*}\left(L \times \mathbb{P}^{1}\right)^{n}\right)-\frac{1}{(n+1) V}\left(\overline{\mathcal{L}}^{n+1}\right) .\right.
\end{aligned}
$$

(iii) The non-Archimedean Ricci energy is

$$
\begin{aligned}
R^{\mathrm{NA}}(\phi): & =V^{-1}\left(\psi_{\text {triv }} \cdot \phi^{n}\right) \\
& =V^{-1}\left(\rho^{*} K_{X \times \mathbb{P}^{1} / \mathbb{P}^{1}}^{\log } \cdot \overline{\mathcal{L}}^{n}\right) .
\end{aligned}
$$

(iv) The non-Archimedean entropy is

$$
\begin{aligned}
H^{\mathrm{NA}}(\phi): & =\int_{X^{\mathrm{div}}} A_{X}(v) \mathrm{MA}^{\mathrm{NA}}(\phi) \\
& =V^{-1}\left(K_{\overline{\mathcal{X}} / \mathbb{P}^{1}}^{\mathrm{log}} \cdot \overline{\mathcal{L}}^{n}\right)-V^{-1}\left(\rho^{*} K_{X \times \mathbb{P}^{1} / \mathbb{P}^{1}}^{\log } \cdot \overline{\mathcal{L}}^{n}\right) .
\end{aligned}
$$


(v) The non-Archimedean Mabuchi functional (or K-energy) is

$$
\begin{aligned}
M^{\mathrm{NA}}(\phi): & =H^{\mathrm{NA}}(\phi)+R^{\mathrm{NA}}(\phi)+\bar{S} E^{\mathrm{NA}}(\phi) \\
& =V^{-1}\left(K_{\overline{\mathcal{X}} / \mathbb{P}^{1}}^{\log } \cdot \overline{\mathcal{L}}^{n}\right)+\frac{\bar{S}}{(n+1) V}\left(\overline{\mathcal{L}}^{n+1}\right) .
\end{aligned}
$$

Note the resemblance to the formulas in \$1.5. All of these functionals are homogeneous. They are also translation invariant, except for $E^{\mathrm{NA}}$ and $R^{\mathrm{NA}}$, which satisfy

$$
E^{\mathrm{NA}}(\phi+c)=E^{\mathrm{NA}}(\phi)+c \text { and } R^{\mathrm{NA}}(\phi+c)=R^{\mathrm{NA}}(\phi)-\bar{S} c
$$

for all $\phi \in \mathcal{H}^{\mathrm{NA}}$ and $c \in \mathbb{Q}$.

The functionals $I^{\mathrm{NA}}, J^{\mathrm{NA}}$ and $I^{\mathrm{NA}}-J^{\mathrm{NA}}$ are comparable on semipositive metrics in the same way as (1.12). By [BHJ15, Lemma 7.7, Theorem 5.16], when $\phi$ is positive, the first term in the definition of $J^{\mathrm{NA}}$ satisfies

$$
V^{-1}\left(\phi \cdot \phi_{\text {triv }}^{n}\right)=\left(\phi-\phi_{\text {triv }}\right)\left(v_{\text {triv }}\right)=\max _{X_{\text {div }}}\left(\phi-\phi_{\text {triv }}\right)=\max _{E} b_{E}^{-1} \operatorname{ord}_{E}(D) .
$$

Further, $J^{\mathrm{NA}}(\phi) \geq 0$, with equality iff $\phi=\phi_{\text {triv }}+c$ for some $c \in \mathbb{Q}$, and $J^{\mathrm{NA}}$ is comparable to both a natural $L^{1}$-norm and the minimum norm in the sense of Dervan [Der15, see [BHJ15, Theorem 7.9, Remark 7.12]. For a normal ample test configuration $(\mathcal{X}, \mathcal{L})$ representing $\phi \in \mathcal{H}^{\mathrm{NA}}$ we also denote the J-norm by $J^{\mathrm{NA}}(\mathcal{X}, \mathcal{L})$.

2.8. The Donaldson-Futaki invariant. As explained in [BHJ15], the non-Archimedean Mabuchi functional is closely related to the Donaldson-Futaki invariant. We have

Proposition 2.8. Assume $L$ is ample. Let $\phi \in \mathcal{H}^{\mathrm{NA}}$ be the class of an ample test configuration $(\mathcal{X}, \mathcal{L})$ for $(X, L)$, and denote by $(\tilde{\mathcal{X}}, \tilde{\mathcal{L}})$ its normalization, which is thus the unique normal, ample representative of $\phi$. Then

$$
\begin{aligned}
M^{\mathrm{NA}}(\phi) & =\operatorname{DF}(\tilde{\mathcal{X}}, \tilde{\mathcal{L}})-V^{-1}\left(\left(\widetilde{\mathcal{X}}_{0}-\widetilde{\mathcal{X}}_{0, \text { red }}\right) \cdot \tilde{\mathcal{L}}^{n}\right) \\
\operatorname{DF}(\mathcal{X}, \mathcal{L}) & =\operatorname{DF}(\tilde{\mathcal{X}}, \tilde{\mathcal{L}})+2 V^{-1} \sum_{E} m_{E}\left(E \cdot \mathcal{L}^{n}\right),
\end{aligned}
$$

where $E$ ranges over the irreducible components of $\mathcal{X}_{0}$ contained in the singular locus of $\mathcal{X}$ and $m_{E} \in \mathbb{N}^{*}$ is the length of $\left(\nu_{*} \mathcal{O}_{\tilde{\mathcal{X}}}\right) / \mathcal{O}_{\mathcal{X}}$ at the generic point of $E$, with $\nu: \widetilde{\mathcal{X}} \rightarrow \mathcal{X}$ the normalization.

In particular, $\operatorname{DF}(\mathcal{X}, \mathcal{L}) \geq M^{\mathrm{NA}}(\phi)$, and equality holds iff $\mathcal{X}$ is regular in codimension one and $\mathcal{X}_{0}$ is generically reduced.

Indeed, (2.2) and (2.3) follow from the discussion in [BHJ15, §7.3] and from [BHJ15, Proposition 3.15], respectively. Note that intersection theoretic formulas for the DonaldsonFutaki invariant appeared already in WWan12] and Oda13.

For a general non-Archimedean metric $\phi$ on $L$ we can define

$$
\begin{aligned}
\operatorname{DF}(\phi) & =M^{\mathrm{NA}}(\phi)+V^{-1}\left(\left(\mathcal{X}_{0}-\mathcal{X}_{0, \text { red }}\right) \cdot \overline{\mathcal{L}}^{n}\right) \\
& =V^{-1}\left(K_{\overline{\mathcal{X}} / \mathbb{P}^{1}} \cdot \overline{\mathcal{L}}^{n}\right)+\frac{\bar{S}}{(n+1) V}\left(\overline{\mathcal{L}}^{n+1}\right)
\end{aligned}
$$

for any normal representative $(\mathcal{X}, \mathcal{L})$ of $\phi$. Clearly $M^{\mathrm{NA}}(\phi) \leq \mathrm{DF}(\phi)$ when $\phi$ is semipositive. 
2.9. The non-Archimedean Ding functional [BHJ15, §7.7]. Suppose $X$ is weakly Fano, that is, $L:=-K_{X}$ is big and nef. In this case, we define the non-Archimedean Ding functional on the space of non-Archimedean metrics on $L$ by

$$
D^{\mathrm{NA}}(\phi)=L^{\mathrm{NA}}(\phi)-E^{\mathrm{NA}}(\phi)
$$

where $L^{\mathrm{NA}}$ is defined by

$$
L^{\mathrm{NA}}(\phi)=\inf _{v}\left(A_{X}(v)+\left(\phi-\phi_{\text {triv }}\right)(v)\right)
$$

the infimum taken over all valuations $v$ on $X$ that are divisorial or trivial. Recall from $\$ 2.5$ that $\phi-\phi_{\text {triv }}$ is a non-Archimedean metric on $\mathcal{O}_{X}$ and induces a bounded function on divisorial valuations. Note that $L^{\mathrm{NA}}(\phi+c)=L^{\mathrm{NA}}(\phi)+c$; hence $D^{\mathrm{NA}}$ is translation invariant.

We always have $D^{\mathrm{NA}} \leq J^{\mathrm{NA}}$, see [BHJ15, Proposition 7.28]. When $\phi$ is semipositive, we have $D^{\mathrm{NA}}(\phi) \leq M^{\mathrm{NA}}(\phi)$, see [BHJ15, Proposition 7.32].

2.10. Uniform K-stability. As in [BHJ15, §8] we make the following definition.

Definition 2.9. A polarized complex manifold $(X, L)$ is uniformly K-stable if there exists a constant $\delta>0$ such that the following equivalent conditions hold.

(i) $M^{\mathrm{NA}}(\phi) \geq \delta J^{\mathrm{NA}}(\phi)$ for every $\phi \in \mathcal{H}^{\mathrm{NA}}(L)$;

(ii) $\mathrm{DF}(\phi) \geq \delta J^{\mathrm{NA}}(\phi)$ for every $\phi \in \mathcal{H}^{\mathrm{NA}}(L)$;

(iii) $\operatorname{DF}(\mathcal{X}, \mathcal{L}) \geq \delta J^{\mathrm{NA}}(\mathcal{X}, \mathcal{L})$ for any normal ample test configuration $(\mathcal{X}, \mathcal{L})$.

Here the equivalence between (ii) and (iii) is definitional, and (i) $\Longrightarrow$ (ii) follows immediately from $\mathrm{DF} \leq M^{\mathrm{NA}}$. The implication (ii) $\Longrightarrow$ (i) follows from the homogeneity of $M^{\mathrm{NA}}$ together with the fact that $\mathrm{DF}\left(\phi_{d}\right)=M^{\mathrm{NA}}\left(\phi_{d}\right)$ for $d$ sufficiently divisible. See [BHJ15, Proposition 8.2] for details.

The fact that $J^{\mathrm{NA}}(\phi)=0$ iff $\phi=\phi_{\text {triv }}+c$ implies that uniform K-stability is stronger than K-stability as introduced by [Tia97, Don02]. Our notion of uniform K-stability is equivalent to uniform K-stability defined either with respect to the $L^{1}$-norm or the minimum norm in the sense of [Der15], see [BHJ15, Remark 8.3].

In the Fano case, uniform K-stability is further equivalent to uniform Ding stability:

Theorem 2.10. Assume $L:=-K_{X}$ is ample and fix a number $\delta$ with $0 \leq \delta \leq 1$. Then the following conditions are equivalent:

(i) $M^{\mathrm{NA}} \geq \delta J^{\mathrm{NA}}$ on $\mathcal{H}^{\mathrm{NA}}$;

(ii) $D^{\mathrm{NA}} \geq \delta J^{\mathrm{NA}}$ on $\mathcal{H}^{\mathrm{NA}}$.

This is proved in [BBJ15] using the Minimal Model Program as in [LX14]. See [Fuj16] for a more general result, and also Fuj15.

\section{Non-Archimedean Limits}

In this section we prove Theorem A and Corollary B. 
3.1. Rays of metrics and non-Archimedean limits. For any line bundle $L$ on $X$, there is a bijection between smooth rays $\left(\phi^{s}\right)_{s>0}$ of metrics on $L$ and $S^{1}$-invariant smooth metrics $\Phi$ on the pull-back of $L$ to $X \times \Delta^{*}$, with $\Delta^{*}=\Delta_{1}^{*} \subset \mathbb{C}$ the punctured unit disc. The restriction of $\Phi$ to $\mathcal{X}_{\tau}$ for $\tau \in \Delta^{*}$ is given by pullback of $\phi^{\log |\tau|^{-1}}$ under the map $\mathcal{X}_{\tau} \rightarrow X$ given by the $\mathbb{C}^{*}$-action. Similarly, smooth rays $\left(\phi^{s}\right)_{s>s_{0}}$ correspond to $S^{1}$-invariant smooth metrics on the pull-back of $L$ to $X \times \Delta_{r_{0}}^{*}$, with $r_{0}=e^{-s_{0}}$.

A subgeodesic ray is a ray $\left(\phi^{s}\right)$ whose corresponding metric $\Phi$ is semipositive. Such rays can of course only exist when $L$ is nef.

Definition 3.1. We say that a smooth ray $\left(\phi^{s}\right)$ admits a non-Archimedean metric $\phi^{\mathrm{NA}}$ as non-Archimedean limit if there exists a test configuration $(\mathcal{X}, \mathcal{L})$ representing $\phi^{\mathrm{NA}}$ such that the metric $\Phi$ on $L \times \Delta^{*}$ corresponding to $\left(\phi^{s}\right)_{s}$ extends to a smooth metric on $\mathcal{L}$ over $\Delta$.

In other words, a non-Archimedean limit exists iff $\Phi$ has analytic singularities along $X \times\{0\}$, i.e. splits into a smooth part and a divisorial part after pulling-back to a blow-up.

Lemma 3.2. Given a ray $\left(\phi^{s}\right)_{s}$ in $\mathcal{H}$, the non-Archimedean limit $\phi^{\mathrm{NA}} \in \mathcal{H}^{\mathrm{NA}}$ is unique, if it exists.

Proof. Let $\psi_{1}$ and $\psi_{2}$ be non-Archimedean limits of $\left(\phi^{s}\right)_{s}$ and let $\Phi$ be the smooth metric on $L \times \Delta^{*}$ defined by the ray $\left(\phi^{s}\right)$. For $i=1,2$, pick a representative $\left(\mathcal{X}_{i}, \mathcal{L}_{i}\right)$ of $\psi_{i}$ such that $\Phi$ extends as a smooth metric on $\mathcal{L}_{i}$ over $\Delta$. After replacing $\left(\mathcal{X}_{i}, \mathcal{L}_{i}\right)$ by suitable pullbacks, we may assume $\mathcal{X}_{1}=\mathcal{X}_{2}=: \mathcal{X}$ and that $\mathcal{X}$ is normal. Then $\mathcal{L}_{2}=\mathcal{L}_{1}+D$ for a $\mathbb{Q}$-divisor $D$ supported on $\mathcal{X}_{0}$. Now a smooth metric on $\mathcal{L}_{1}$ induces a singular metric on $\mathcal{L}_{1}+D$ that is smooth iff $D=0$. Hence $\mathcal{L}_{1}=\mathcal{L}_{2}$, so that $\psi_{1}=\psi_{2}$.

Remark 3.3. Following [Berk09, §2] (see also [Jon16, BJ16a]) one can construct a compact Hausdorff space $X^{\mathrm{An}}$ fibering over the interval $[0,1]$ such that the fiber $X_{\rho}^{\mathrm{An}}$ over any point $\rho \in(0,1]$ is homeomorphic to the complex manifold $X$, and the fiber $X_{0}^{\mathrm{An}}$ over 0 is homeomorphic to the Berkovich analytification of $X$ with respect to the trivial norm on $\mathbb{C}$. Similarly, the line bundle $L$ induces a line bundle $L^{\mathrm{An}}$ over $X^{\mathrm{An}}$. If a ray $\left(\phi^{s}\right)_{s>0}$ admits a non-Archimedean limit $\phi^{\mathrm{NA}}$, then it induces a continuous metric on $L^{\mathrm{An}}$ whose restriction to $L_{\rho}^{\mathrm{An}}$ is given by $\phi^{\log \rho^{-1}}$ and whose restriction to $X_{0}^{\mathrm{an}}$ is given by $\phi^{\mathrm{NA}}$. In this way, $\phi^{\mathrm{NA}}$ is indeed the limit of $\phi^{s}$ as $s \rightarrow \infty$.

3.2. Non-Archimedean limits of functionals. For the rest of 33 , assume that $L$ is ample.

Definition 3.4. A functional $F: \mathcal{H} \rightarrow \mathbb{R}$ admits a functional $F^{\mathrm{NA}}: \mathcal{H}^{\mathrm{NA}} \rightarrow \mathbb{R}$ as a non-Archimedean limit if, for every smooth subgeodesic ray $\left(\phi^{s}\right)$ in $\mathcal{H}$ admitting a nonArchimedean limit $\phi^{\mathrm{NA}} \in \mathcal{H}^{\mathrm{NA}}$, we have

$$
\lim _{s \rightarrow+\infty} \frac{F\left(\phi^{s}\right)}{s}=F^{\mathrm{NA}}\left(\phi^{\mathrm{NA}}\right) .
$$

Proposition 3.5. If $F: \mathcal{H} \rightarrow \mathbb{R}$ admits a non-Archimedean limit $F^{\mathrm{NA}}: \mathcal{H}^{\mathrm{NA}} \rightarrow \mathbb{R}$, then $F^{\mathrm{NA}}$ is homogeneous.

Proof. Consider a semiample test configuration $(\mathcal{X}, \mathcal{L})$ representing a non-Archimedean metric $\phi^{\mathrm{NA}} \in \mathcal{H}^{\mathrm{NA}}$, and let $\left(\phi^{s}\right)_{s}$ be a smooth subgeodesic ray admitting $\phi^{\mathrm{NA}}$ as a nonArchimedean limit. For $d \geq 1$, let $\left(\mathcal{X}_{d}, \mathcal{L}_{d}\right)$ be the normalized base change induced by $\tau \rightarrow \tau^{d}$. The associated non-Archimedean metric $\phi_{d}^{\mathrm{NA}}$ is then the non-Archimedean limit 
of the subgeodesic ray $\left(\phi^{d s}\right)$, so $\lim _{s \rightarrow \infty} s^{-1} F\left(\phi_{d s}\right)=F^{\mathrm{NA}}\left(\phi_{d}^{\mathrm{NA}}\right)$. On the other hand, we clearly have $\lim _{s \rightarrow \infty}(d s)^{-1} F\left(\phi^{d s}\right)=\lim _{s \rightarrow \infty} s^{-1} F\left(\phi^{s}\right)=F^{\mathrm{NA}}\left(\phi^{\mathrm{NA}}\right)$. The result follows.

3.3. Asymptotics of the functionals. The following result immediately implies Theorem A and Corollary B.

Theorem 3.6. The functionals $E, H, I, J, M$ and $R$ on $\mathcal{H}$ admit non-Archimedean limits on $\mathcal{H}^{\mathrm{NA}}$ given, respectively, by $E^{\mathrm{NA}}, H^{\mathrm{NA}}, I^{\mathrm{NA}}, J^{\mathrm{NA}}, M^{\mathrm{NA}}$ and $R^{\mathrm{NA}}$.

In addition, we have the following result due to Berman [Berm16, Proposition 3.8]. See also [BBJ15, Theorem 3.1] for a more general result.

Theorem 3.7. If $L:=-K_{X}$ is ample, then the Ding functional $D$ on $\mathcal{H}$ admits $D^{\mathrm{NA}}$ on $\mathcal{H}^{\mathrm{NA}}$ as non-Archimedean limit.

Remark 3.8. In $\$$ we will extend the two previous results to the logarithmic setting and with relaxed positivity assumptions.

The main tool in the proof of Theorem 3.6 is the following result (compare [PRS08, Lemma 6]).

Lemma 3.9. For $i=0, \ldots, n$, let $L_{i}$ be a line bundle on $X$ with a smooth reference metric $\phi_{i, \mathrm{ref}}$. Let also $\left(\mathcal{X}, \mathcal{L}_{i}\right)$ be a smooth test configuration for $\left(X, L_{i}\right), \Phi_{i}$ an $S^{1}$-invariant smooth metric on $\mathcal{L}_{i}$ near $\mathcal{X}_{0}$, and denote by $\left(\phi_{i}^{s}\right)$ the corresponding ray of smooth metrics on $L_{i}$. Then

$$
\left\langle\phi_{0}^{s}, \ldots, \phi_{n}^{s}\right\rangle_{X}-\left\langle\phi_{0, \text { ref }}, \ldots, \phi_{n, \text { ref }}\right\rangle_{X}=s\left(\overline{\mathcal{L}}_{0} \cdot \ldots \cdot \overline{\mathcal{L}}_{n}\right)+O(1)
$$

as $s \rightarrow \infty$. Here $\left(\overline{\mathcal{X}}, \overline{\mathcal{L}}_{i}\right)$ is the compactification of $\left(\mathcal{X}, \mathcal{L}_{i}\right)$ for $0 \leq i \leq n$ and $\langle\cdot, \ldots, \cdot\rangle_{X}$ denotes the Deligne pairing with respect to the constant morphism $X \rightarrow\{\mathrm{pt}\}$.

Proof. The Deligne pairing $F:=\left\langle\mathcal{L}_{0}, \ldots, \mathcal{L}_{n}\right\rangle_{\mathcal{X} / \mathbb{C}}$ is a line bundle on $\mathbb{C}$, endowed with a $\mathbb{C}^{*}$ action and a canonical identification of its fiber at $\tau=1$ with the complex line $\left\langle L_{0}, \ldots, L_{n}\right\rangle_{X}$. It extends to a line bundle $\left\langle\overline{\mathcal{L}}_{0}, \ldots, \overline{\mathcal{L}}_{n}\right\rangle_{\overline{\mathcal{X}} / \mathbb{P}^{1}}$ on $\mathbb{P}^{1}$ that is $\mathbb{C}^{*}$-equivariantly trivial on $\mathbb{P}^{1} \backslash\{0\}$. Denoting by $w \in \mathbb{Z}$ the weight of the $\mathbb{C}^{*}$-action on the fiber at 0 , we have

$$
w=\operatorname{deg}\left\langle\overline{\mathcal{L}}_{0}, \ldots, \overline{\mathcal{L}}_{n}\right\rangle_{\overline{\mathcal{X}} / \mathbb{P}^{1}}=\left(\overline{\mathcal{L}}_{0}, \ldots, \overline{\mathcal{L}}_{n}\right) .
$$

Pick a nonzero vector $v \in F_{1}=\left\langle L_{0}, \ldots, L_{n}\right\rangle_{X}$. The $\mathbb{C}^{*}$-action produces a section $\tau \mapsto \tau \cdot v$ of $F$ on $\mathbb{C}^{*}$, and $\sigma:=\tau^{-w}(\tau \cdot v)$ is a nowhere vanishing section of $F$ on $\mathbb{C}$, see BHJ15, Corollary 1.4].

Since the metrics $\Phi_{i}$ are smooth and $S^{1}$-invariant, $\Psi:=\left\langle\Phi_{0}, \ldots, \Phi_{n}\right\rangle_{\mathcal{X} / \mathbb{C}}$ is a continuous $S^{1}$-invariant metric on $F$ near $0 \in \mathbb{C}$. Hence the function $\log |\sigma|_{\Psi}$ is bounded near $0 \in \mathbb{C}$.

The $S^{1}$-invariant metric $\Psi$ defines a ray $\left(\psi^{s}\right)$ of metrics on the line $F_{1}$ through $|v|_{\psi^{s}}=$ $|\tau \cdot v|_{\Psi_{\tau}}$, for $s=\log |\tau|^{-1}$, where $\Psi_{\tau}$ is the restriction of $\Psi$ to $F_{\tau}$. Thus

$$
\log |v|_{\psi^{s}}=\log |\tau \cdot v|_{\Psi_{\tau}}=w \log |\tau|+\log |\sigma|_{\Psi_{\tau}}=-s w+O(1) .
$$

By functoriality, the metric $\psi^{s}$ on $F_{1}$ is nothing but the Deligne pairing $\left\langle\phi_{0}^{s}, \ldots, \phi_{n}^{s}\right\rangle$. If we set $\psi_{\text {ref }}=\left\langle\phi_{0, \text { ref }}, \ldots, \phi_{n, \text { ref }}\right\rangle_{X}$, it therefore follows that

$$
\left\langle\phi_{0}^{s}, \ldots, \phi_{n}^{s}\right\rangle_{X}-\left\langle\phi_{0, \text { ref }}, \ldots, \phi_{n, \text { ref }}\right\rangle_{X}=\log |v|_{\psi_{\text {ref }}}-\log |v|_{\psi^{s}}=s w+O(1)
$$

which completes the proof. 
Proof of Theorem 3.6. Let $\left(\phi^{s}\right)_{s}$ be a smooth subgeodesic ray in $\mathcal{H}$ admitting a non-Archimedean limit $\phi^{\mathrm{NA}} \in \mathcal{H}^{\mathrm{NA}}$. Pick a test configuration $(\mathcal{X}, \mathcal{L})$ representing $\phi^{\mathrm{NA}}$ such that $\mathcal{X}$ is smooth and $\mathcal{X}_{0}$ has snc support. Thus $\mathcal{L}$ is relatively semiample and $\left(\phi^{s}\right)_{s}$ corresponds to a smooth $S^{1}$-invariant semipositive metric $\Phi$ on $\mathcal{L}$ over $\Delta$. By Lemma 1.2 , we have

$$
(n+1) V\left(E\left(\phi^{s}\right)-E\left(\phi_{\text {ref }}\right)\right)=\left\langle\phi^{s}, \ldots, \phi^{s}\right\rangle_{X}-\left\langle\phi_{\text {ref }}, \ldots, \phi_{\text {ref }}\right\rangle_{X} .
$$

Using Lemma 3.9, it follows that

$$
\lim _{s \rightarrow+\infty} \frac{E\left(\phi^{s}\right)}{s}=\frac{\left(\overline{\mathcal{L}}^{n+1}\right)}{(n+1) V}=E^{\mathrm{NA}}\left(\phi^{\mathrm{NA}}\right),
$$

which proves the result for the Monge-Ampère energy $E$. The case of the functionals $I, J$ and $R$ is similarly a direct consequence of Lemma 1.2 and Lemma 3.9. In view of the ChenTian formulas for $M$ and $M^{\mathrm{NA}}$, it remains to consider the case of the entropy functional $H$. In fact, it turns out to be easier to treat the functional $H+R$.

By Lemma 1.2 we have

$$
V\left(H\left(\phi^{s}\right)+R\left(\phi^{s}\right)\right)=\left\langle\frac{1}{2} \log \mathrm{MA}\left(\phi^{s}\right), \phi^{s}, \ldots, \phi^{s}\right\rangle_{X}-\left\langle\psi_{\text {ref }}, \phi_{\text {ref }}, \ldots, \phi_{\text {ref }}\right\rangle_{X},
$$

where $\psi_{\text {ref }}=\frac{1}{2} \log \mathrm{MA}\left(\phi_{\text {ref }}\right)$, so we must show that

$$
\left\langle\frac{1}{2} \log \operatorname{MA}\left(\phi^{s}\right), \phi^{s}, \ldots, \phi^{s}\right\rangle_{X}-\left\langle\psi_{\text {ref }}, \phi_{\text {ref }}, \ldots, \phi_{\text {ref }}\right\rangle_{X}=s\left(K_{\overline{\mathcal{X}} / \mathbb{P}^{1}}^{\log } \cdot \overline{\mathcal{L}}^{n}\right)+o(s) .
$$

The collection of metrics $\frac{1}{2} \log \operatorname{MA}\left(\left.\Phi\right|_{\mathcal{X}_{\tau}}\right)$ with $\tau \neq 0$ defines a smooth metric $\Psi$ on $K_{\mathcal{X} / \mathbb{C}}^{\log }$ over $\Delta^{*}$, but the difficulty here (as opposed to the situation in [PRS08]) is that $\Psi$ will not a priori extend to a smooth (or even locally bounded) metric on $K_{\mathcal{X} / \mathbb{C}}^{\log }$ over $\Delta$. Indeed, since we have assumed that $\mathcal{X}$ is smooth, there is no reason why $\Phi$ is strictly positive.

Instead, pick a smooth, $S^{1}$-invariant reference metric $\Psi_{\text {ref }}$ on $K_{\mathcal{X} / \mathbb{C}}^{\log }$ over $\Delta$, and denote by $\left(\psi_{\text {ref }}^{s}\right)_{s>0}$ the corresponding ray of smooth metrics on $K_{X}$. By Lemma 3.9 we have

$$
\left\langle\psi_{\text {ref }}^{s}, \phi^{s}, \ldots, \phi^{s}\right\rangle_{X}-\left\langle\psi_{\text {ref }}, \phi_{\text {ref }}, \ldots, \phi_{\text {ref }}\right\rangle_{X}=s\left(K_{\overline{\mathcal{X}} / \mathbb{P}^{1}}^{\log } \cdot \overline{\mathcal{L}}^{n}\right)+O(1) .
$$

Since

$$
\left\langle\frac{1}{2} \log \operatorname{MA}\left(\phi^{s}\right), \phi^{s}, \ldots, \phi^{s}\right\rangle_{X}-\left\langle\psi_{\mathrm{ref}}^{s}, \phi^{s}, \ldots, \phi^{s}\right\rangle_{X}=\frac{1}{2} \int_{X} \log \left[\frac{\mathrm{MA}\left(\phi^{s}\right)}{e^{2 \psi_{\mathrm{ref}}^{s}}}\right]\left(d d^{c} \phi^{s}\right)^{n},
$$

Theorem 3.6 is therefore a consequence of the following result.

Lemma 3.10. We have $\int_{X} \log \left[\frac{\mathrm{MA}\left(\phi^{s}\right)}{e^{2 \psi_{\mathrm{ref}}^{s}}}\right]\left(d d^{c} \phi^{s}\right)^{n}=O(\log s)$ as $s \rightarrow \infty$.

Let us first prove an estimate of independent interest. See [BJ16a] for more precise results.

Lemma 3.11. Let $\mathcal{X}$ be an snc test configuration for $X$ and $\Psi$ a smooth metric on $K_{\mathcal{X} / \mathbb{C}}^{\log }$ near $\mathcal{X}_{0}$. Denote by $e^{2 \Psi_{\tau}}$ the induced volume form on $\mathcal{X}_{\tau}$ for $\tau \neq 0$. Then

$$
\int_{\mathcal{X}_{\tau}} e^{2 \Psi_{\tau}} \sim\left(\log |\tau|^{-1}\right)^{d} \quad \text { as } \tau \rightarrow 0
$$

with $d$ denoting the dimension of the dual complex of $\mathcal{X}_{0}$, so that $d+1$ is the largest number of local components of $\mathcal{X}_{0}$.

Here $A \sim B$ means that $A / B$ is bounded from above and below by positive constants. 
Proof. Since $\mathcal{X}_{0}$ is an snc divisor, every point of $\mathcal{X}_{0}$ admits local coordinates $\left(z_{0}, \ldots, z_{n}\right)$ that are defined in a neighborhood of $B:=\left\{\left|z_{i}\right| \leq 1\right\}$ and such that $z_{0}^{b_{0}} \ldots z_{p}^{b_{p}}=\varepsilon \tau$ with $0 \leq p \leq n$ and $\varepsilon>0$. Here $b_{i} \in \mathbb{Z}_{>0}$ is the multiplicity of $\mathcal{X}_{0}$ along $\left\{z_{i}=0\right\}$. The integer $d$ in the statement of the theorem is then the largest such integer $p$. By compactness of $\mathcal{X}_{0}$, it will be enough to show that

$$
\int_{B \cap \mathcal{X}_{\tau}} e^{2 \Psi_{\tau}} \sim\left(\log |\tau|^{-1}\right)^{p}
$$

The holomorphic $n$-form

$$
\eta:=\frac{1}{p+1} \sum_{j=0}^{p} \frac{(-1)^{j}}{b_{j}} \frac{d z_{0}}{z_{0}} \wedge \cdots \wedge \frac{\widehat{d z_{j}}}{z_{j}} \wedge \cdots \wedge \frac{d z_{p}}{z_{p}} \wedge d z_{p+1} \wedge \cdots \wedge d z_{n}
$$

satisfies

$$
\eta \wedge \frac{d \tau}{\tau}=\frac{d z_{0}}{z_{0}} \wedge \cdots \wedge \frac{d z_{p}}{z_{p}} \wedge d z_{p+1} \wedge \cdots \wedge d z_{n}
$$

Thus $\eta$ defines a local frame of $K_{\mathcal{X} / \mathbb{C}}^{\log }$ on $B$, so the holomorphic $n$-form $\eta_{\tau}:=\left.\eta\right|_{\mathcal{X}_{\tau}}$ satisfies

$$
C^{-1}\left|\eta_{\tau}\right|^{2} \leq e^{2 \Psi_{\tau}} \leq C\left|\eta_{\tau}\right|^{2}
$$

for a constant $C>0$ independent of $\tau$. Hence it suffices to prove $\int_{B \cap \mathcal{X}_{\tau}}\left|\eta_{\tau}\right|^{2} \sim\left(\log |\tau|^{-1}\right)^{p}$.

To this end, we parametrize $B \cap \mathcal{X}_{\tau}$ in (logarithmic) polar coordinates as follows. Consider the $p$-dimensional simplex

$$
\sigma=\left\{w \in \mathbb{R}_{\geq 0}^{p+1} \mid \sum_{j=0}^{p} b_{j} w_{j}=1\right\},
$$

the $p$-dimensional (possibly disconnected) commutative compact Lie group

$$
T=\left\{\theta \in(\mathbb{R} / \mathbb{Z})^{p+1} \mid \sum_{j=0}^{p} b_{j} \theta_{j}=0\right\},
$$

and the polydisc $\mathbb{D}^{n-p} \subset \mathbb{C}^{n-p}$. We may cover $\mathbb{C}^{*}$ by two simply connected open sets, on each of which we fix a branch of the complex logarithm. We then define a diffeomorphism $\chi_{\tau}$ from $\sigma \times T \times \mathbb{D}^{n-p}$ to $B \cap \mathcal{X}_{\tau}$ by setting

$$
z_{j}=e^{w_{j} \log (\varepsilon \tau)+2 \pi i \theta_{j}} \quad \text { for } 0 \leq j \leq p .
$$

A simple computation shows that

$$
\chi_{\tau}^{*}\left(\left|\eta_{\tau}\right|^{2}\right)=\operatorname{const}\left(\log |\varepsilon \tau|^{-1}\right)^{p} d V,
$$

where $d V$ denotes the natural volume form on $\sigma \times T \times \mathbb{D}^{n-p}$. It follows that, for $|\tau| \ll 1$,

$$
\int_{B \cap \mathcal{X}_{\tau}}\left|\eta_{\tau}\right|^{2} \sim \int_{\sigma \times T \times \mathbb{D}^{n-p}} \chi_{\tau}^{*}\left(\left|\eta_{\tau}\right|^{2}\right) \sim\left(\log |\tau|^{-1}\right)^{p},
$$

which completes the proof. 
Proof of Lemma 3.10. On the one hand, we have

$$
\begin{aligned}
V^{-1} \int_{X} \log \left[\frac{\mathrm{MA}\left(\phi^{s}\right)}{e^{2 \psi_{\text {ref }}^{s}}}\right]\left(d d^{c} \phi^{s}\right)^{n} \\
\quad=\int_{X} \log \left[\frac{\mathrm{MA}\left(\phi^{s}\right)}{e^{2 \psi_{\text {ref }}^{s}} / \int_{X} e^{2 \psi_{\text {ref }}^{s}}}\right] \operatorname{MA}\left(\phi^{s}\right)-\log \int_{X} e^{2 \psi_{\text {ref }}^{s}} \geq-\log \int_{X} e^{2 \psi_{\text {ref }}^{s},}
\end{aligned}
$$

since the first term on the second line is the relative entropy of the probability measure $\operatorname{MA}\left(\phi^{s}\right)$ with respect to the probability measure $e^{2 \psi_{\text {ref }}^{s}} / \int_{X} e^{2 \psi_{\text {ref }}^{s}}$. By Lemma 3.11 we have $\int_{X} e^{2 \psi_{\text {ref }}^{s}}=O\left(s^{d}\right)$, where $0 \leq d \leq n$. This gives the lower bound in Lemma 3.10 .

To get the upper bound, it suffices to prove that the function $g_{\tau}:=\frac{\left(d d^{c} \Phi \mid \mathcal{X}_{\tau}\right)^{n}}{e^{2 \Psi_{\tau}}}$ on $\mathcal{X}_{\tau}$ is uniformly bounded from above. Indeed, if $\tau=e^{-s}$, we then see that

$$
\int_{X} \log \left[\frac{\mathrm{MA}\left(\phi^{s}\right)}{e^{2 \psi_{\text {ref }}^{s}}}\right]\left(d d^{c} \phi^{s}\right)^{n}=\int_{\mathcal{X}_{\tau}}\left(\log V^{-1}+\log g_{\tau}\right)\left(\left.d d^{c} \Phi\right|_{\mathcal{X}_{\tau}}\right)^{n}
$$

is uniformly bounded from above, since $\left(\left.d d^{c} \Phi\right|_{\mathcal{X}_{\tau}}\right)^{n}$ has fixed mass $V$ for all $\tau$.

To bound $g_{\tau}$ from above, we use local coordinates $\left(z_{j}\right)_{0}^{n}$ as in the proof of Lemma 3.11 . With the notation in that proof, it suffices to prove that the function $\left(\left.\Omega\right|_{\mathcal{X}}\right)^{n} / e^{2 \Psi_{\tau}}$ on $\mathcal{X}_{\tau}$ is uniformly bounded from above, where $\Omega:=\frac{i}{2} \sum_{j=0}^{n} d z_{j} \wedge d \bar{z}_{j}$. Indeed, we have $d d^{c} \Phi \leq C \Omega$ for some constant $C>0$. It then further suffices to prove the bound

$$
\left.i^{n} d z_{0} \wedge d \bar{z}_{0} \wedge \cdots \wedge d \widehat{z_{j} \wedge d} \bar{z}_{j} \wedge \cdots \wedge d z_{n} \wedge d z_{n}\right|_{\mathcal{X}_{\tau}} \leq C e^{2 \Psi_{\tau}}
$$

for $0 \leq j \leq p$ and a uniform constant $C>0$.

To prove (3.4) we use the logarithmic polar coordinates in the proof of Lemma 3.10 . Namely, if $\chi_{\tau}: \sigma \times T \times \mathbb{D}^{n-p} \rightarrow B \cap X_{\tau}$ is the diffeomorphism in that proof, we have

$$
\begin{gathered}
\chi_{\tau}^{*}\left(e^{2 \Psi_{\tau}}\right) \sim\left(\log |\tau|^{-1}\right)^{p} d V . \\
\chi_{\tau}^{*}\left(i^{n} d z_{0} \wedge d \bar{z}_{0} \wedge \cdots \wedge d \widehat{z_{j} \wedge d} \bar{z}_{j} \wedge \cdots \wedge d z_{n} \wedge d z_{n}\right) \sim\left(\log |\tau|^{-1}\right)^{p} \prod_{0 \leq l \leq p, l \neq j}\left|z_{l}\right|^{2} d V .
\end{gathered}
$$

Thus (3.4) holds, which completes the proof.

\section{The LOGARIThmic Setting}

In this section we extend, for completeness, Theorem 3.6 - and hence Theorem A and Corollary B - to the logarithmic setting. We will also relax the positivity assumptions used. Our conventions and notation largely follow [BBEGZ11].

4.1. Preliminaries. If $X$ is a normal projective variety of dimension $n$, and $\phi_{1}, \ldots, \phi_{n}$ are smooth metrics on $\mathbb{Q}$-line bundles $L_{1}, \ldots, L_{n}$ on $X$, then we define $d d^{c} \phi_{1} \wedge \cdots \wedge d d^{c} \phi_{n}$ as the pushforward of the measure $\left.\left.d d^{c} \phi_{1}\right|_{X_{\mathrm{reg}}} \wedge \cdots \wedge d d^{c} \phi_{n}\right|_{X_{\mathrm{reg}}}$ from $X_{\text {reg }}$ to $X$. This is a signed Radon measure of total mass $\left(L_{1} \cdot \ldots \cdot L_{n}\right)$, positive if the $\phi_{i}$ are semipositive.

A boundary on $X$ is a Weil $\mathbb{Q}$-divisor $B$ on $X$ such that the Weil $\mathbb{Q}$-divisor class

$$
K_{(X, B)}:=K_{X}+B
$$

is $\mathbb{Q}$-Cartier. Note that $B$ is not necessarily effective. We call $(X, B)$ a pair. 
The log discrepancy of a divisorial valuation $v=c \operatorname{ord}_{F}$ with respect to $(X, B)$ is defined as in $\$ 2.3$, using $A_{(X, B)}(v)=c\left(1+\operatorname{ord}_{F}\left(K_{Y /(X, B)}\right)\right)$. The pair $(X, B)$ is subklt if $A_{(X, B)}(v)>0$ for all (nontrivial) divisorial valuations $v$. (It is klt when $B$ is further effective.)

A pair $(X, B)$ is $\log$ smooth if $X$ is smooth and $B$ has simple normal crossing support. A $\log$ resolution of $(X, B)$ is a projective birational morphism $f: X^{\prime} \rightarrow X$, with $X^{\prime}$ smooth, such that $\operatorname{Exc}(f)+f_{*}^{-1}(B)$ has simple normal crossing support. In this case, there is a unique snc divisor $B^{\prime}$ on $X^{\prime}$ such that $f_{*} B^{\prime}=B$ and $K_{\left(X^{\prime}, B^{\prime}\right)}=f^{*} K_{(X, B)}$. In particular the pair $\left(X^{\prime}, B^{\prime}\right)$ is log smooth. The pair $(X, B)$ is subklt iff $\left(X^{\prime}, B^{\prime}\right)$ is subklt, and the latter is equivalent to $B^{\prime}$ having coefficients $<1$.

A smooth metric $\psi$ on $K_{(X, B)}$ canonically defines a smooth positive measure $\mu_{\psi}$ on $X_{\text {reg }} \backslash B$ as follows. Let $\phi_{B}$ be the canonical singular metric on $\mathcal{O}_{X_{\text {reg }}}(B)$, with curvature current given by $[B]$. Then $\psi-\phi_{B}$ is a smooth metric on $K_{X_{\mathrm{reg}} \backslash B}$, and hence induces a smooth positive measure

$$
\mu_{\psi}:=e^{2\left(\psi-\phi_{B}\right)}
$$

on $X_{\text {reg }} \backslash B$. The fact that $(X, B)$ is subklt means precisely that the total mass of $\mu_{\psi}$ is finite. Thus we can view $\mu_{\psi}$ as a finite positive measure on $X$ that is smooth on $X_{\text {reg }} \backslash B$ and gives no mass to $B$ or $X_{\text {sing }}$.

4.2. Archimedean functionals. Let $X$ be a normal complex projective variety of dimension $n$. Fix a big and nef $\mathbb{Q}$-line bundle $L$ on $X$ and set $V:=\left(L^{n}\right)>0$. For a smooth metric $\phi$ on $L$, set $\mathrm{MA}(\phi)=V^{-1}\left(d d^{c} \phi\right)^{n}$.

Fix a smooth positive reference metric $\phi_{\text {ref }}$ on $L$ The energy functionals $E, I$ and $J$ are defined on smooth metrics on $L$ exactly as in (1.6), (1.8) and (1.7), respectively; they are normalized by $E\left(\phi_{\text {ref }}\right)=I\left(\phi_{\text {ref }}\right)=J\left(\phi_{\text {ref }}\right)=0$. The functionals $I$ and $J$ are translation invariant, whereas $E(\phi+c)=E(\phi)+c$. All three functionals are pullback invariant in the following sense. Let $q: X^{\prime} \rightarrow X$ be a birational morphism, with $X^{\prime}$ normal and projective, and set $L^{\prime}:=q^{*} L$. For any smooth metric $\phi$ on $L$, we have $E\left(\phi^{\prime}\right)=E(\phi), I\left(\phi^{\prime}\right)=I(\phi)$ and $J\left(\phi^{\prime}\right)=J(\phi)$, where $\phi^{\prime}=q^{*} \phi$ and where the functionals are computed with respect to the reference metric $\phi_{\text {ref }}^{\prime}:=q^{*} \phi_{\text {ref }}$.

Now consider a boundary $B$ on $X$. Set $\bar{S}_{B}:=-n V^{-1}\left(K_{(X, B)} \cdot L^{n-1}\right)$ and fix a smooth reference metric $\psi_{\text {ref }}$ on $K_{(X, B)}$. When $X$ is smooth and $B=0$, we could pick $\psi_{\text {ref }}=$ $\frac{1}{2} \log \mathrm{MA}\left(\phi_{\text {ref }}\right)$, but in general, there seems to be no canonical way to get $\psi_{\text {ref }}$ from $\phi_{\text {ref }}$.

The analogue of the Ricci energy $R$ is defined on smooth metrics $\phi$ on $L$ by

$$
R_{B}(\phi):=\sum_{j=0}^{n-1} \frac{1}{V} \int_{X_{\mathrm{reg}}}\left(\phi-\phi_{\mathrm{ref}}\right) d d^{c} \psi_{\mathrm{ref}} \wedge\left(d d^{c} \phi\right)^{j} \wedge\left(d d^{c} \phi_{\mathrm{ref}}\right)^{n-1-j} .
$$

It satisfies $R_{B}(\phi+c)=R_{B}(\phi)-\bar{S}_{B} c$ and is pullback invariant in the following sense. Suppose $q: X^{\prime} \rightarrow X$ is a birational morphism, with $X^{\prime}$ projective normal, and define $B^{\prime}$ by $q_{*} B^{\prime}=B$ and $q^{*} K_{(X, B)}=K_{\left(X^{\prime}, B^{\prime}\right)}$. Set $\phi_{\text {ref }}^{\prime}=q^{*} \phi_{\text {ref }}$ and $\psi_{\text {ref }}^{\prime}:=q^{*} \psi_{\text {ref }}$. Then $R_{B}(\phi)=R_{B^{\prime}}\left(\phi^{\prime}\right)$, where $\phi^{\prime}=q^{*} \phi$.

Now assume $(X, B)$ is subklt and let $\mu_{\text {ref }}=\mu_{\psi_{\text {ref }}}$ be the finite positive measure defined in 4.1 . It is smooth and positive on $X_{\text {ref }} \backslash B$, and may be assumed to have mass 1 , after adding a constant to $\psi_{\text {ref }}$. For a smooth semipositive metric $\phi$ on $L$, set

$$
H_{B}(\phi):=\frac{1}{2} \int_{X_{\mathrm{reg}}} \log \frac{\mathrm{MA}(\phi)}{\mu_{\mathrm{ref}}} \operatorname{MA}(\phi)=\frac{1}{2} \int_{X_{\mathrm{reg}}} \log \frac{\mathrm{MA}(\phi)}{e^{2\left(\psi_{\mathrm{ref}}-\phi_{B}\right)}} \mathrm{MA}(\phi) .
$$


We may have $H_{B}\left(\phi_{\text {ref }}\right) \neq 0$. However, $H_{B}$ is bounded from below and translation invariant. It is also pullback invariant in the sense above, with reference measure $\mu_{\text {ref }}^{\prime}=\mu_{\psi_{\text {ref }}^{\prime}}$ on $X^{\prime}$.

Lemma 4.1. If $\phi$ is a smooth semipositive metric on $L$, then $H_{B}(\phi)<+\infty$.

Proof. By pullback invariance we may assume that $(X, B)$ is $\log$ smooth. In this case $\mathrm{MA}(\phi)$ and $\mu_{\text {ref }}$ are smooth measures on $X$ that are strictly positive on $X_{\text {reg }}$. Consider any point $\xi \in B$ and pick local coordinates $\left(z_{1}, \ldots, z_{n}\right)$ at $\xi$ such that the irreducible components of $B$ are given by $\left\{z_{i}=0\right\}, 0 \leq i \leq p$. Fix a volume form $d V$ near $\xi$. Then $\mu_{\text {ref }}=g \prod_{i=0}^{p}\left|z_{i}\right|^{2 a_{i}} d V$, and $\operatorname{MA}(\phi)=h d V$, with $a_{i}>-1, g>0$ and $h \geq 0$ smooth. If $f=h \log \left(\frac{h}{g} \prod_{i=0}^{p}\left|z_{i}\right|^{-2 a_{i}}\right)$, then $f$ is locally integrable with respect to $d V$. This completes the proof.

As in 1.4 we define the Mabuchi functional on semipositive smooth metrics by

$$
M_{B}:=H_{B}+R_{B}+\bar{S}_{B} E .
$$

Then $M_{B}$ is translation invariant and pullback invariant in the sense above. At least formally, the critical points of $M_{B}$ satisfy

$$
n\left(\operatorname{Ric}\left(d d^{c} \phi\right)-[B]\right) \wedge\left(d d^{c} \phi\right)^{n-1}=\bar{S}_{B}\left(d d^{c} \phi\right)^{n}
$$

and should be conical cscK metrics, see [Li14].

Finally consider the (weak) $\log$ Fano case, in which $L:=-K_{(X, B)}$ is big and nef. The Ding functional is then defined on smooth metrics as $D_{B}=L_{B}-E$, with

$$
L_{B}(\phi):=-\frac{1}{2} \log \int_{X_{\mathrm{reg}}} e^{-2\left(\phi+\phi_{B}\right)}
$$

If we use $\psi_{\text {ref }}=-\phi_{\text {ref }}$, then the formula for the Mabuchi functional simplifies to

$$
M_{B}(\phi)=H_{B}(\phi)-\left(E(\phi)-\int_{X_{\text {reg }}}\left(\phi-\phi_{\text {ref }}\right) \mathrm{MA}(\phi)\right) .
$$

We have $D_{B} \leq M_{B}$ on smooth semipositive metrics.

4.3. Non-Archimedean functionals. The extensions of the non-Archimedean functionals in $\$ 2.7$ to the logarithmic setting were studied in [BHJ15, §7]. Let us briefly review them.

Consider a normal complex projective variety $X$ and a big and nef $\mathbb{Q}$-line bundle $L$ on $X$. Let $\phi$ be a non-Archimedean metric on $L$, represented by a normal test configuration $(\mathcal{X}, \mathcal{L})$ for $(X, L)$, that we assume dominates $(X \times \mathbb{C}, L \times \mathbb{C})$ via $\rho: \mathcal{X} \rightarrow X \times \mathbb{C}$. The formulas in 2.7 for $E^{\mathrm{NA}}(\phi), I^{\mathrm{NA}}(\phi)$ and $J^{\mathrm{NA}}(\phi)$ are still valid.

Given a boundary $B$ on $X$ we set

$$
\begin{aligned}
R_{B}^{\mathrm{NA}}(\phi): & =V^{-1}\left(\psi_{\text {triv }} \cdot \phi^{n}\right) \\
& =V^{-1}\left(\rho^{*} K_{\left(X \times \mathbb{P}^{1}, B \times \mathbb{P}^{1}\right) / \mathbb{P}^{1}}^{\log } \cdot \overline{\mathcal{L}}^{n}\right) .
\end{aligned}
$$

Now assume $(X, B)$ is subklt and let $\mathcal{B}$ (resp. $\overline{\mathcal{B}}$ ) be the (component wise) Zariski closure of $B \times \mathbb{C}^{*}$ in $\mathcal{X}(\operatorname{resp} . \overline{\mathcal{X}})$. Then

$$
\begin{aligned}
H_{B}^{\mathrm{NA}}(\phi): & =\int_{X^{\mathrm{div}}} A_{(X, B)}(v) \mathrm{MA}^{\mathrm{NA}}(\phi) \\
& =V^{-1}\left(K_{(\overline{\mathcal{X}}, \overline{\mathcal{B}}) / \mathbb{P}^{1}}^{\log } \cdot \overline{\mathcal{L}}^{n}\right)-V^{-1}\left(\rho^{*} K_{\left(X \times \mathbb{P}^{1}, B \times \mathbb{P}^{1}\right) / \mathbb{P}^{1}}^{\log } \cdot \overline{\mathcal{L}}^{n}\right) .
\end{aligned}
$$


and

$$
\begin{aligned}
M_{B}^{\mathrm{NA}}(\phi): & =H_{B}^{\mathrm{NA}}(\phi)+R_{B}^{\mathrm{NA}}(\phi)+\bar{S}_{B} E^{\mathrm{NA}}(\phi) \\
& =\frac{1}{V}\left(K_{(\overline{\mathcal{X}}, \overline{\mathcal{B}}) / \mathbb{P}^{1}}^{\log } \cdot \overline{\mathcal{L}}^{n}\right)+\frac{\bar{S}_{B}}{(n+1) V}\left(\overline{\mathcal{L}}^{n+1}\right) .
\end{aligned}
$$

While the definitions of $H_{B}^{\mathrm{NA}}(\phi)$ and $M_{B}^{\mathrm{NA}}(\phi)$ make sense for arbitrary non-Archimedean metrics $\phi$, we will usually assume that $\phi$ is semipositive.

All the functionals above have the same invariance properties as their Archimedean cousins. They are also homogeneous in the sense of Definition 2.7.

Finally, when $(X, B)$ is weakly $\log$ Fano, so that $(X, B)$ is subklt and $L:=-K_{(X, B)}$ is big and nef, the non-Archimedean Ding functional is defined by

$$
D_{B}^{\mathrm{NA}}(\phi)=L_{B}^{\mathrm{NA}}(\phi)-E^{\mathrm{NA}}(\phi),
$$

where

$$
L_{B}^{\mathrm{NA}}(\phi)=\inf _{v}\left(A_{(X, B)}(v)+\left(\phi-\phi_{\text {triv }}\right)(v)\right),
$$

the infimum taken over all valuations $v$ on $X$ that are divisorial or trivial.

The Ding functional $D_{B}^{\mathrm{NA}}$ is translation invariant and pullback invariant. The formula for the Mabuchi functional simplifies in the log Fano case to

$$
M_{B}^{\mathrm{NA}}(\phi)=H_{B}^{\mathrm{NA}}(\phi)-\left(E^{\mathrm{NA}}(\phi)-\int_{X_{\text {div }}}\left(\phi-\phi_{\mathrm{ref}}\right) \mathrm{MA}^{\mathrm{NA}}(\phi)\right) .
$$

We have $D_{B}^{\mathrm{NA}} \leq \min \left\{M_{B}^{\mathrm{NA}}, J^{\mathrm{NA}}\right\}$ on semipositive metrics, see Propositions 7.28 and 7.32 in [BHJ15].

4.4. Asymptotics. The following result generalizes Theorem 3.6 and shows that if $F$ is one of the functionals $E, I, J, H_{B}, R_{B}$ or $M_{B}$ on $\mathcal{H}$, then $F$ admits a non-Archimedean limit on $\mathcal{H}^{\mathrm{NA}}$ given by $F^{\mathrm{NA}}$. For future reference, we state the result in detail.

Theorem 4.2. Let $X$ be a normal projective variety, $L$ a big and nef $\mathbb{Q}$-line bundle on $X$, and $(\mathcal{X}, \mathcal{L})$ a test configuration for $(X, L)$ inducing a non-Archimedean metric $\phi^{\mathrm{NA}}$ on L. Further, let $\Phi$ be a smooth, $S^{1}$-invariant metric on $\mathcal{L}$ near $\mathcal{X}_{0}$, inducing a smooth ray $\left(\phi^{s}\right)_{s>s_{0}}$ of metrics on L. Fix a smooth reference metric $\phi_{\text {ref }}$ on L. Then

$$
\lim _{s \rightarrow+\infty} \frac{F\left(\phi^{s}\right)}{s}=F^{\mathrm{NA}}\left(\phi^{\mathrm{NA}}\right),
$$

where $F$ is any of the functionals $E, I, J$.

Further, if $B$ is a boundary on $X$ and $\psi_{\mathrm{ref}}$ is a smooth reference metric on $K_{(X, B)}$, then (4.1) also holds for $F=R_{B}$. Finally, if $(X, B)$ is subklt and $\Phi$ is semipositive, then (4.1) holds for $F=H_{B}$ and $F=M_{B}$.

In addition, Berman proved that in the log Fano case, the Ding functional $D_{B}$ admits $D_{B}^{\mathrm{NA}}$ as non-Archimedean limit. Indeed, the following result follows from Proposition 3.8 and $\S 4.3$ in [Berm16].

Theorem 4.3. Let $(X, B)$ be a subklt pair with $L:=-K_{(X, B)}$ big and nef, $(\mathcal{X}, \mathcal{L})$ a test configuration for $(X, L)$ inducing a non-Archimedean metric $\phi^{\mathrm{NA}}$ on $L$, and $\Phi$ a semipositive smooth, $S^{1}$-invariant metric on $\mathcal{L}$ near $\mathcal{X}_{0}$, inducing a smooth ray $\left(\phi^{s}\right)_{s>s_{0}}$ of semipositive metrics on L. Then $\lim _{s \rightarrow+\infty} \frac{1}{s} D_{B}\left(\phi^{s}\right)=D_{B}^{\mathrm{NA}}\left(\phi^{\mathrm{NA}}\right)$. 
In fact, it is enough to assume $\Phi$ is semipositive and locally bounded in Theorem 4.3.

Remark 4.4. Theorems 4.2 and 4.3 remain true even when $\Phi$ is not $S^{1}$-invariant, in the following sense. For $\tau \in \Delta^{*}$, let $\phi_{\tau}$ be the metric on $L$ defined as the pullback of $\left.\Phi\right|_{\mathcal{X}_{\tau}}$ under the $\mathbb{C}^{*}$-action. Then we have $\lim _{\tau \rightarrow 0}\left(\log |\tau|^{-1}\right)^{-1} F\left(\phi_{\tau}\right)=F^{\mathrm{NA}}\left(\phi^{\mathrm{NA}}\right)$.

4.5. Proof of Theorem 4.2. By pullback invariance, we may assume that $X$ is smooth. After further pullback, we may also assume that $\mathcal{X}$ is smooth and dominates $X \times \mathbb{C}$. In this case, the asymptotic formulas for $E, I$ and $J$ follow immediately from Lemma 3.9 .

When considering the remaining functionals, we may similarly, by pullback invariance, assume that the pair $(X, B)$ is $\log$ smooth. The asymptotic formula for $R_{B}$ now follows from Lemma 3.9 since we can express $R_{B}(\phi)$ in terms of Deligne pairings:

$$
R_{B}(\phi)=\left\langle\psi_{\text {ref }}, \phi^{n}\right\rangle_{X}-\left\langle\psi_{\text {ref }}, \phi_{\text {ref }}^{n}\right\rangle_{X}
$$

whereas the non-Archimedean counterpart is given by the intersection number

$$
R_{B}^{\mathrm{NA}}(\phi)=V^{-1}\left(\rho^{*} K_{\left(X \times \mathbb{P}^{1}, B \times \mathbb{P}^{1}\right) / \mathbb{P}^{1}}^{\log } \cdot \overline{\mathcal{L}}^{n}\right)_{\overline{\mathcal{X}}} .
$$

Finally we consider the functionals $H_{B}$ and $M_{B}$. Thus assume $(X, B)$ is log smooth and subklt. We may further assume that the divisor $\mathcal{X}_{0}+\mathcal{B}$ has simple normal crossing support, where $\mathcal{B}$ is the (component-wise) Zariski closure of the pullback of $B \times \mathbb{C}^{*}$ in $\mathcal{X}$.

As in 3.3 it suffices to prove the asymptotic formula for the functional $H_{B}+R_{B}$. To this end, we express $H_{B}$ in terms of Deligne pairings. Write $B=\sum_{i} c_{i} B_{i}$, where $B_{i}, i \in I$, are the irreducible components of $B$ and $c_{i} \in \mathbb{Q}$. Fix a smooth metric $\psi_{i}$ on $\mathcal{O}_{X}\left(B_{i}\right)$ for $i \in I$. Then $\psi_{B}:=\sum_{i} c_{i} \psi_{i}$ is a smooth metric on $\mathcal{O}_{X}(B)$, and it follows from 1.3 that

$$
\begin{aligned}
V H_{B}(\phi) & =\frac{1}{2} \int_{X} \log \frac{\mathrm{MA}(\phi)}{e^{2\left(\psi_{\mathrm{ref}}-\psi_{B}\right)}}\left(d d^{c} \phi\right)^{n}+\sum_{i \in I} c_{i} \int_{X} \log \left|\sigma_{i}\right|_{\psi_{i}}\left(d d^{c} \phi\right)^{n} \\
& =\left\langle\frac{1}{2} \log \operatorname{MA}(\phi), \phi^{n}\right\rangle_{X}-\left\langle\psi_{\mathrm{ref}}, \phi^{n}\right\rangle_{X}+\left\langle\psi_{B}, \phi^{n}\right\rangle_{X}+\sum_{i \in I} c_{i}\left(\left\langle\phi^{n}\right\rangle_{B_{i}}-\left\langle\psi_{i}, \phi^{n}\right\rangle_{X}\right) \\
& =\left\langle\frac{1}{2} \log \operatorname{MA}(\phi), \phi^{n}\right\rangle_{X}-\left\langle\psi_{\mathrm{ref}}, \phi^{n}\right\rangle_{X}+\sum_{i \in I} c_{i}\left\langle\phi^{n}\right\rangle_{B_{i}},
\end{aligned}
$$

for any smooth semipositive metric $\phi$ on $L$. This implies

$$
\begin{aligned}
V\left(H_{B}(\phi)+R_{B}(\phi)\right) & =\left\langle\frac{1}{2} \log \operatorname{MA}(\phi), \phi^{n}\right\rangle_{X}-\left\langle\psi_{\text {ref }}, \phi_{\text {ref }}^{n}\right\rangle_{X}+\sum_{i \in I} c_{i}\left\langle\phi^{n}\right\rangle_{B_{i}} \\
& =V(H(\phi)+R(\phi))+n \sum_{i \in I} c_{i}\left(L^{n-1} \cdot B_{i}\right) E\left(\left.\phi\right|_{B_{i}}\right)+O(1) .
\end{aligned}
$$

On the non-Archimedean side, we have

$$
\begin{aligned}
V\left(H_{B}^{\mathrm{NA}}\left(\phi^{\mathrm{NA}}\right)+R_{B}^{\mathrm{NA}}\left(\phi^{\mathrm{NA}}\right)\right) & =\left(K_{(\overline{\mathcal{X}}, \overline{\mathcal{B}}) / \mathbb{P}^{1}}^{\log } \cdot \overline{\mathcal{L}}^{n}\right)_{\overline{\mathcal{X}}} \\
& =\left(K_{\overline{\mathcal{X}} / \mathbb{P}^{1}}^{\log } \cdot \overline{\mathcal{L}}^{n}\right)_{\overline{\mathcal{X}}}+\left(\overline{\mathcal{B}} \cdot \overline{\mathcal{L}}^{n}\right)_{\overline{\mathcal{X}}} \\
& =V\left(H^{\mathrm{NA}}\left(\phi^{\mathrm{NA}}\right)+R^{\mathrm{NA}}\left(\phi^{\mathrm{NA}}\right)\right)+\sum_{i \in I} c_{i}\left(\left.\overline{\mathcal{L}}\right|_{\overline{\mathcal{B}}_{i}} ^{n}\right)_{\overline{\mathcal{B}}_{i}} \\
& =V\left(H^{\mathrm{NA}}\left(\phi^{\mathrm{NA}}\right)+R^{\mathrm{NA}}\left(\phi^{\mathrm{NA}}\right)\right)+n \sum_{i \in I} c_{i}\left(L^{n-1} \cdot B_{i}\right) E^{\mathrm{NA}}\left(\phi_{i}^{\mathrm{NA}}\right)
\end{aligned}
$$


where $\phi_{i}^{\mathrm{NA}}$ is the non-Archimedean metric on $\left.L\right|_{B_{i}}$ represented by $\left.\mathcal{L}\right|_{\mathcal{B}_{i}}$.

It now follows from Theorem 3.6 that 1

$$
\lim _{s \rightarrow \infty} \frac{1}{s}\left(H\left(\phi^{s}\right)+R\left(\phi^{s}\right)\right)=H^{\mathrm{NA}}\left(\phi^{\mathrm{NA}}\right)+R\left(\phi^{\mathrm{NA}}\right),
$$

Applying Theorem 3.6 on $B_{i}$ and $\mathcal{B}_{i}$, we also get $\lim _{s \rightarrow \infty} \frac{1}{s} E\left(\phi_{i}^{s}\right)=E^{\mathrm{NA}}\left(\phi_{i}^{\mathrm{NA}}\right)$. Thus

$$
\lim _{s \rightarrow \infty} \frac{1}{s}\left(H_{B}\left(\phi^{s}\right)+R_{B}\left(\phi^{s}\right)\right)=H_{B}^{\mathrm{NA}}\left(\phi^{\mathrm{NA}}\right)+R_{B}\left(\phi^{\mathrm{NA}}\right),
$$

which completes the proof of Theorem 4.2 .

4.6. Coercivity and uniform K-stability. Let us finally extend Corollary B to the logarithmic setting. Consider a pair $(X, B)$ and a big and nef line bundle $L$ on $X$. The Donaldson-Futaki invariant of a normal test configuration $(\mathcal{X}, \mathcal{L})$ for $(X, L)$ is given by

$$
\begin{aligned}
\mathrm{DF}_{B}(\mathcal{X}, \mathcal{L}): & =\frac{1}{V}\left(K_{(\overline{\mathcal{X}} \cdot \overline{\mathcal{B}}) / \mathbb{P}^{1}} \cdot \overline{\mathcal{L}}^{n}\right)+\bar{S}_{B} \frac{\left(\overline{\mathcal{L}}^{n+1}\right)}{(n+1) V} \\
& =M_{B}^{\mathrm{NA}}(\phi)+\frac{1}{V}\left(\left(\mathcal{X}_{0}-\mathcal{X}_{0, \text { red }}\right) \cdot \mathcal{L}^{n}\right),
\end{aligned}
$$

where $\phi$ is the non-Archimedean metric on $L$ represented by $\phi$. Now assume $L$ is ample. We then define $(X, B) ; L)$ to be uniformly $K$-stable if the following two equivalent conditions hold:

(i) there exists $\delta>0$ such that $M_{B}^{\mathrm{NA}}(\phi) \geq \delta J^{\mathrm{NA}}(\phi)$ for every $\phi \in \mathcal{H}^{\mathrm{NA}}(L)$;

(ii) there exists $\delta>0$ such that $\mathrm{DF}_{B}(\mathcal{X}, \mathcal{L}) \geq \delta J^{\mathrm{NA}}(\mathcal{X}, \mathcal{L})$ for any normal ample test configuration $(\mathcal{X}, \mathcal{L})$.

The equivalence between the two conditions is proved in [BHJ15, Proposition 8.2].

Corollary 4.5. Let $(X, B)$ be a subklt pair and $L$ an ample line bundle on $X$. Suppose that the Mabuchi functional is coercive in the sense that there exist positive constants $\delta$ and $C$ such that $M_{B}(\phi) \geq \delta J(\phi)-C$ for every positive smooth metric $\phi$ on $L$. Then $((X, B) ; L)$ is uniformly K-stable; more precisely $\mathrm{DF}_{B}(\mathcal{X}, \mathcal{L}) \geq M_{B}(\phi) \geq \delta J^{\mathrm{NA}}(\phi)$ for every positive nonArchimedean metric on $L$, where $(\mathcal{X}, \mathcal{L})$ is the unique normal ample representative of $\phi$.

\section{UnifORM K-STABILITY AND CM-STABILITY}

From now on, $X$ is smooth. In this section we explore the relationship between uniform K-stability and (asymptotic) CM-stability. In particular we prove Theorem C, Corollary D and Corollary E.

5.1. Functions with log norm singularities. In this section, $G$ denotes a reductive complex algebraic group.

Definition 5.1. We say that a function $f: G \rightarrow \mathbb{R}$ has $\log$ norm singularities if there exist finitely many rational numbers $a_{i}$, finite dimensional complex vector spaces $V_{i}$ endowed with an algebraic $G$-action and non-zero vectors $v_{i} \in V_{i}$ such that

$$
f(g)=\sum_{i} a_{i} \log \left\|g \cdot v_{i}\right\|+O(1)
$$

\footnotetext{
${ }^{1}$ While Theorem 3.6 is stated in the case when $L$ and $\mathcal{L}$ are ample and $\Phi$ is positive, the proof extends to the weaker positivity assumptions used here.
} 
for some choice of norms on the $V_{i}$ 's.

Remark 5.2. By the equivalence of norms on a finite dimensional vector space, the description of $f$ is independent of the choice of norms on the $V_{i}$. In particular, given a maximal compact subgroup $K$ of $G$, the norms may be assumed to be $K$-invariant, so that $f$ descends to a function on the Riemannian symmetric space $G / K$.

Remark 5.3. Taking appropriate tensor products, is is easy to see that every function $f$ on $G$ with log norm singularities may be written as

$$
f(g)=a(\log \|g \cdot v\|-\log \|g \cdot w\|)+O(1),
$$

where $a \in \mathbb{Q}_{>0}$ and $v, w$ are vectors in a normed vector space $V$ endowed with a $G$-action.

An algebraic group homomorphism $\lambda: \mathbb{C}^{*} \rightarrow G$ is called a one-parameter subgroup (1-PS for short). The following generalization of the Kempf-Ness/Hilbert-Mumford criterion is closely related to Pau13. Our argument, which is based on Mumford's original proof of the Hilbert-Mumford criterion [MFF, §2.1], fixes in particular the proof of [Pau13, Theorem 4.2], as well as an incorrect argument provided in a previous version of the present paper.

Theorem 5.4. Let $f$ be a function on $G$ with log norm singularities.

(i) For each 1-PS $\lambda: \mathbb{C}^{*} \rightarrow G$, there exists $f^{\mathrm{NA}}(\lambda) \in \mathbb{Q}$ such that

$$
(f \circ \lambda)(\tau)=f^{\mathrm{NA}}(\lambda) \log |\tau|^{-1}+O(1)
$$

for $|\tau| \leq 1$.

(ii) The function $f$ is bounded below on $G$ iff $f^{\mathrm{NA}}(\lambda) \geq 0$ for all 1-PS $\lambda$.

The chosen notation stems from the fact that $f^{\mathrm{NA}}$ induces a function on the (conical) Tits building of $G$, i.e. the non-Archimedean analogue of $G / K$ (compare [MFF, §2.2]).

By Remark 5.3 we may and do assume that $f$ is of the form

$$
f(g):=\log \|g \cdot v\|-\log \|g \cdot w\|,
$$

where $v, w$ are nonzero vectors in a finite dimensional normed vector space $V$ equipped with a linear $G$-action. In that case, the following variant of the Kempf-Ness criterion, observed in [Pau13, Proposition 4], translates Theorem 5.4 into an algebro-geometric statement.

Lemma 5.5. The function $f(g)=\log \|g \cdot v\|-\log \|g \cdot w\|$ is bounded below on $G$ if and only if the Zariski closure of the orbit of $[v, w] \in \mathbb{P}(V \oplus W)$ does not intersect the subspace $\mathbb{P}(\{0\} \oplus W)$.

Proof. As with any algebraic group action, the orbit $G \cdot[v, w]$ is a complex algebraic subvariety of $\mathbb{P}(V \oplus W)$, i.e. a locally closed subset in the Zariski topology. Its Zariski closure therefore coincides with its closure in the Euclidean topology, and the argument is then elementary. Indeed, assume $f\left(g_{i}\right) \rightarrow-\infty$ for some sequence $g_{i} \in G$, i.e. $\left\|g_{i} \cdot v\right\|=o\left(\left\|g_{i} \cdot w\right\|\right)$. After passing to a subsequence, $\tilde{w}_{i}:=\left(g_{i} \cdot w\right) /\left\|g_{i} \cdot w\right\|$ converges (in the Euclidian topology) to a nonzero vector in $W$, while $\tilde{v}_{i}:=\left(g_{i} \cdot v\right) /\left\|g_{i} \cdot w\right\|$ tends to 0 ; hence $g_{i} \cdot[v, w] \in G \cdot[v, w]$ converges to $[0, \tilde{w}] \in \mathbb{P}(\{0\} \oplus W)$. Conversely, if $g_{i} \cdot[v, w] \rightarrow[0, \tilde{w}]$ for some sequence $g_{i} \in G$ and nonzero $\tilde{w} \in W$, then $c_{i}\left(g_{i} \cdot v\right) \rightarrow 0$ in $V$ and $c_{i}\left(g_{i} \cdot w\right) \rightarrow \tilde{w}$ in $W$ with $c_{i} \in \mathbb{C}^{*}$, and hence $f\left(g_{i}\right)=\log \left\|c_{i}\left(g_{i} \cdot v\right)\right\|-\log \left\|c_{i}\left(g_{i} \cdot w\right)\right\| \rightarrow-\infty$. 
The key ingredient in the proof of Theorem 5.4 is the following algebro-geometric result, which will be obtained as a consequence of the Iwahori decomposition theorem, very much as in $\mathrm{MFF}$.

Theorem 5.6. Let $G$ be a complex reductive group with a linear action on a finite dimensional complex vector space $U$. If the (Zariski) closure of the $G$-orbit of a point $x \in \mathbb{P}(U)$ meets a $G$-invariant Zariski closed subset $Z \subset \mathbb{P}(U)$, then some $z \in Z \cap \overline{G \cdot x}$ can be reached by a 1-PS $\lambda$ of $G$, i.e. $\lim _{\tau \rightarrow 0} \lambda(\tau) \cdot x=z$.

Remark 5.7. As explained in [Don12, $\S 5]$, it is however not true in general that any $z \in$ $Z \cap \overline{G \cdot x}$ can be reached by a 1-PS $\lambda$, unless the stabilizer of $z$ in $G$ is reductive.

Introduce the formal power series ring $R=\mathbb{C} \llbracket t \rrbracket$ and its fraction field $K:=\mathbb{C}((t))$, and let $X$ be a complex algebraic variety. Viewed as a $\mathbb{C}$-scheme, $X$ is separated, and the set of $R$ points $X(R)$, i.e. morphisms $\gamma: \operatorname{Spec} R \rightarrow X$ over Spec $\mathbb{C}$, thus injects into $X(K)$. Further, each $\gamma \in X(R)$ admits a reduction $\tilde{\gamma} \in X(\mathbb{C})$. If $X$ is proper (i.e. $X(\mathbb{C})$ is compact), the valuative criterion yields $X(R)=X(K)$, which means that any 'meromorphic arc' $\gamma:$ Spec $K \rightarrow X$ uniquely extends across the closed point of $\operatorname{Spec} R$, whose image is $\tilde{\gamma}$. In case $X=\mathbb{P}(U)$ for a complex vector space $U$, this becomes very concrete: for each $\gamma \in X(K)=\mathbb{P}\left(U_{K}\right)$, there exists $u \in U_{R}$, unique up to multiplication by a unit of $R$, such that $\gamma=[u]$ and $\tilde{u} \neq 0$ in $U$, and we then have $\tilde{\gamma}=[\tilde{u}]$.

The following valuative criterion was used without precise reference in Mumford's proof of the Hilbert-Mumford criterion [MFF, p.54]. We provide here some details (see [Ant, §4] for a closely related discussion).

Lemma 5.8. Let $\phi: Y \rightarrow X$ be a morphism between complex algebraic varieties, and let $x \in X(\mathbb{C})$ be a closed point. Then $x$ belongs to the (Zariski) closure of the image $\phi(Y)$ if and only if there exists $\gamma \in Y(K)$ with $\phi(\gamma) \in X(R)$ and $\widetilde{\phi(\gamma)}=x$.

Proof. The condition is clearly sufficient. Assume conversely that $x$ is in the Zariski closure of $\phi(Y)$. Replacing $X$ with the closure of $\phi(Y)$, we may assume that $\phi$ is dominant. By Chevalley's theorem, $\phi(Y)$ is constructible, i.e. a finite union of locally closed subsets; being dense in $X$, it thus contains a non-empty open subset $U \subset X$. Using for instance Noether normalization, it is easy to construct a closed point $p \in C$ on a smooth algebraic curve and a morphism $f: C \rightarrow X$ with $f(p)=x$ and $f^{-1}(U)$ non-empty [Kem, Lemma 7.2.1]. It follows that the restriction of the induced morphism $\operatorname{Spec} \mathcal{O}_{C, p} \rightarrow X$ to the generic point lifts to $Y$, and passing to the formal completion of $C$ at $p$ yields the result.

Proof of Theorem 5.6. The action of $G$ on $X:=\mathbb{P}(U)$, being algebraic, induces an action of the group $G(K)$ on the set $X(K)$. Since $K$ is an extension of $\mathbb{C}$, the closed point $x \in X$ can be viewed as an element of $X(K)$, and our goal is to find a point $\lambda \in G(K)$ corresponding to a one-parameter subgroup of $G$ such that the reduction of $\lambda \cdot x \in X(K)$ belong to $Z$.

Given any 1-PS $\lambda \in G(K)$ and $\xi \in X(K)$, we first claim that the reduction of $\lambda \cdot \xi \in X(K)$ only depends on $\tilde{\xi} \in X(\mathbb{C})$. Indeed, denote by $U=\bigoplus_{m \in \mathbb{Z}} U_{m}$ the weight decomposition with respect to $\lambda$. As mentioned above, there exists $u \in U_{R}$, unique up to a unit in $R$, such 
that $\xi=[u]$ and $\tilde{u} \neq 0$. The reduction of

$$
\lambda \cdot \xi=\left[\sum_{m} t^{m} u_{m}\right]
$$

is equal to $\left[\widetilde{u_{p}}\right]$ with $p:=\min \left\{m \mid \widetilde{u_{m}} \neq 0\right\}$, and hence only depends on $\tilde{\xi}=[\tilde{u}]$.

Example 5.9. This claim is incorrect, as illustrated by the following counterexample kindly communicated to us by Yan Li. Consider $\xi:=[1: 0], \xi^{\prime}:=[1: t]$ in $\mathbb{P}^{1}(K)$ and the $1-P S \lambda:=\operatorname{diag}\left(t^{2}, t^{-2}\right)$. Then $\tilde{\xi}=\tilde{\xi}^{\prime}=[1: 0] \in \mathbb{P}^{1}(\mathbb{C})$, but $\lambda \cdot \xi=\left[t^{2}: 0\right]=[1: 0]$, $\lambda \cdot \xi^{\prime}=\left[t^{2}: t^{-1}\right]=\left[t^{3}: 1\right]$, and hence $\widetilde{\lambda \cdot \xi}=[1: 0] \neq[0: 1]=\widetilde{\lambda \cdot \xi^{\prime}}$.

Now let $\phi: G \rightarrow X$ be the orbit morphism $\phi(g)=g \cdot x$. By assumption, $\phi(G)$ contains a closed point $z \in Z$ in its Zariski closure, and Lemma 5.8 thus implies the existence of $\gamma \in G(K)$ such that the reduction of $\phi(\gamma)=\gamma \cdot x \in X(K)$ is equal to $z$.

By Iwahori's theorem (cf. [MFF, p.52]), we can find a decomposition $\gamma=\alpha \lambda \beta$ in $G(K)$ with $\alpha, \beta \in G(R)$ and $\lambda \in G(K)$ induced by a 1-PS. By $G$-invariance of $Z$, the reduction of $(\lambda \beta) \cdot x$ belongs to $Z$. After replacing $\lambda$ with $\tilde{\beta} \lambda \tilde{\beta}^{-1}$ and $\beta$ with $\tilde{\beta}^{-1} \beta$, we may assume that $\tilde{\beta}=e \in G(\mathbb{C})$. As a result, $\tilde{x}=\widetilde{\beta \cdot x}$, and the above claim implies that $\widetilde{\lambda \cdot x}=\widetilde{(\lambda \beta) \cdot x}$ belongs to $Z$.

Proof of Theorem 5.4. (i) Let $\lambda: \mathbb{C}^{*} \rightarrow G$ be a 1-parameter subgroup, and denote by $V=$ $\bigoplus_{m \in \mathbb{Z}} V_{m}$ the corresponding weight decompositon. For $\tau \in \mathbb{C}^{*}$, we then have

$$
\lambda(\tau) \cdot v=\sum_{m} \tau^{m} v_{m}
$$

and hence

$$
\log \|\lambda(\tau) \cdot v\|=\max _{v_{m} \neq 0}\left(m \log |\tau|+\log \left\|v_{m}\right\|\right)+O(1)=-\left(\min _{v_{m} \neq 0} m\right) \log |\tau|^{-1}+O(1)
$$

for $|\tau| \leq 1$. This proves (i) with with $f^{\mathrm{NA}}(\lambda)=\min \left\{m \mid w_{m} \neq 0\right\}-\min \left\{m \mid v_{m} \neq 0\right\}$.

(ii) By (i), $f^{\mathrm{NA}}(\lambda) \geq 0$ for all 1-PS $\lambda$ if and only if $f \circ \lambda$ is bounded below on $\mathbb{C}^{*}$ for all $\lambda$. By Lemma 5.5, $f \circ \lambda$ is bounded below on $\mathbb{C}^{*}$ iff $\lim _{\tau \rightarrow 0} \lambda(\tau) \cdot[v, w]$ does not belong to the $G$-invariant Zariski closed subset $Z:=\mathbb{P}(\{0\} \oplus W)$, while $f$ is bounded below on $G$ iff $Z \cap \overline{G \cdot[v, w]}=\emptyset$. The equivalence now follows from Theorem 5.6 .

5.2. Proof of Theorem $\mathbf{C}$ and Corollaries $\mathbf{D}$ and E. Replacing $L$ with $m L$, we may assume for notational simplicity that $m=1$. Set $N:=h^{0}(L)$ and $G:=\operatorname{SL}(N, \mathbb{C})$, so that each $\sigma \in G$ defines a Fubini-Study type metric $\phi_{\sigma}$ on $L$. Note that $M-\delta J$ is bounded below on $\mathcal{H}_{1} \simeq \mathrm{GL}(N, \mathbb{C}) / \mathrm{U}(N)$ iff $M\left(\phi_{\sigma}\right)-\delta J\left(\phi_{\sigma}\right)$ bounded below for $\sigma \in G$, by translation invariance of $M$ and $J$.

The key ingredient is the following result of S. Paul [Pau12] (see also Kap13)

Theorem 5.10. The functionals $E, J$ and $M$ all have $\log$ norm singularities on $G$.

Granted this result we can deduce Theorem C. The equivalence of (ii) and (iii) follows from the same argument as Proposition 8.2 in [BHJ15, so it suffices to show that (i) and (iii) 
are equivalent. By Theorem 5.10, the function $f(\sigma):=M\left(\phi_{\sigma}\right)-\delta J\left(\phi_{\sigma}\right)$ on $G$ has log norm singularities. By Theorem 5.4 it is thus bounded below iff

$$
\lim _{s \rightarrow+\infty} \frac{(f \circ \lambda)\left(e^{-s}\right)}{s} \geq 0
$$

for each 1-parameter subgroup $\lambda: \mathbb{C}^{*} \rightarrow G$. We obtain the desired result since by Theorem B, this limit is equal to $M^{\mathrm{NA}}\left(\phi_{\lambda}\right)-\delta J^{\mathrm{NA}}\left(\phi_{\lambda}\right)$, where $\phi_{\lambda} \in \mathcal{H}^{\mathrm{NA}}$ is the non-Archimedean metric on $L$ defined by $\lambda$.

Corollary $\mathrm{D}$ follows since every ample test configuration of $(X, L)$ is induced by a 1-PS, see $\$ 2.2$ The first assertion of Corollary E follows immediately, and the fact that the reduced automorphism group of $(X, L)$ is finite is a consequence of [Pau13, Corollary 1.1].

Proof of Theorem 5.10, Recall from Pau12] that to the linearly normal embedding $X \hookrightarrow$ $\mathbb{P} H^{0}(X, L)^{*} \simeq \mathbb{P}^{N-1}$ are associated the $X$-resultant $R$, i.e. the Chow coordinate of $X$, and the $X$-hyperdiscriminant $\Delta$, which cuts out the dual variety of

$$
X \times \mathbb{P}^{n-1} \hookrightarrow \mathbb{P}^{N-1} \times \mathbb{P}^{n-1} \hookrightarrow \mathbb{P}^{N n-1}
$$

the second arrow being the Segre embedding.

In our notation, we then have $\operatorname{deg} R=V(n+1)$ and $\operatorname{deg} \Delta=V(n(n+1)-\bar{S})$ Pau12, Proposition 5.7], and [Pau12, Theorem A] becomes

$$
M\left(\phi_{\sigma}\right)=V^{-1} \log \|\sigma \cdot \Delta\|-V^{-1} \frac{\operatorname{deg} \Delta}{\operatorname{deg} R} \log \|\sigma \cdot R\|+O(1),
$$

which proves the assertion for $M\left(\phi_{\sigma}\right)$.

We next consider

$$
J\left(\phi_{\sigma}\right)=\int_{X}\left(\phi_{\sigma}-\phi_{\text {ref }}\right) \mathrm{MA}\left(\phi_{\text {ref }}\right)-E\left(\phi_{\sigma}\right) .
$$

On the one hand, by [Pau04, Theorem 1] (or [Zha96, Theorem 1.6, Theorem 3.6]) we have

$$
E\left(\phi_{\sigma}\right)=\frac{1}{\operatorname{deg} R} \log \|\sigma \cdot R\|+O(1) .
$$

On the other hand, choosing any norm on the space of complex $N \times N$-matrices (in which $G$ of course embeds), it is observed in the proof of [Tia17, Lemma 3.2] that

$$
\int_{X}\left(\phi_{\sigma}-\phi_{\text {ref }}\right) \operatorname{MA}\left(\phi_{\text {ref }}\right)=\log \|\sigma\|+O(1) \text {. }
$$

The assertion for $J\left(\phi_{\sigma}\right)$ follows.

5.3. Discussion of [Tia17]. The statement of [Tia17, Lemma 3.1] sounds overoptimistic from the GIT point of view, as it would mean that CM-stability can be tested by only considering 1-parameter subgroups of a fixed maximal torus $T$.

At least, the proof is incorrect, the problem being the estimate (3.1), which claims that $\phi_{\tau k}-\phi_{\tau}$ is uniformly bounded with respect to $\tau \in T$ and $k \in K$. As the next example shows, this is not even true for a fixed $k \in K$.

Example 5.11. Assume $\left(s_{1}, s_{2}\right)$ is a basis of $H^{0}(X, L)$, let $k \in U(2)$ be the unitary transformation exchanging $s_{1}$ and $s_{2}, \tau=\left(t, t^{-1}\right)$, and pick a point $x$ with $s_{1}(x)=0$. Then

$$
\phi_{\tau k}(x)-\phi_{\tau}(x)=4 \log |\tau|
$$

is unbounded. 
In any case, the methods here do not seem to be able to deduce CM-stability from Kstability, because of the following fact (cf. [Li12, p.39]).

Proposition 5.12. For each polarized manifold $(X, L)$ and each $m$ large and divisible enough, there exists a non-trivial 1-PS $\lambda$ in $\operatorname{GL}\left(N_{m}, \mathbb{C}\right)$ such that $J$ and $M$ remain bounded on the corresponding Fubini-Study ray $\phi^{s}:=\phi_{\lambda\left(e^{-s}\right)}$.

Proof. As originally observed in [LX14] (cf. Proposition 2.3), $(X, L)$ admits a non-trivial ample test configuration $(\mathcal{X}, \mathcal{L})$ that is almost trivial, i.e. with trivial normalization. As recalled in 22.2 , for each $m$ large and divisible enough, $(\mathcal{X}, \mathcal{L})$ can be realized as the test configuration induced by a 1 -PS $\lambda: \mathbb{C}^{*} \rightarrow \operatorname{GL}\left(N_{m}, \mathbb{C}\right)$, which is non-trivial since $(\mathcal{X}, \mathcal{L})$ is. Since the normalization of $(\mathcal{X}, \mathcal{L})$ is trivial, the associated non-Archimedean metric is of the form $\phi_{\text {triv }}+c$ for some $c \in \mathbb{Q}$, and hence $M^{\mathrm{NA}}\left(\phi_{\lambda}\right)=J^{\mathrm{NA}}\left(\phi_{\lambda}\right)=0$. Since $M$ and $J$ have log norm singularities on $\mathrm{GL}\left(N_{m}, \mathbb{C}\right)$ by Theorem 5.10, $M$ and $J$ are indeed bounded on $\phi^{s}$ by Theorem 5.4 .

\section{Remarks on the Yau-Tian-Donaldson conjecture}

As explained in the introduction, we will here give a simple argument, following ideas of Tian, for the existence of a Kähler-Einstein metric on a Fano manifold $X$, assuming $\left(X,-K_{X}\right)$ is uniformly K-stable and the partial $C^{0}$-estimates due to Székelyhidi.

6.1. Partial $C^{0}$-estimates and the continuity method. For the moment, consider an arbitrary polarized manifold $(X, L)$. For each $m$ such that $m L$ is very ample, we have a 'Bergman kernel approximation' map $P_{m}: \mathcal{H} \rightarrow \mathcal{H}_{m}$, defined by setting $P_{m}(\phi)$ to be the Fubini-Study metric induced by the $L^{2}$-scalar product on $H^{0}(X, m L)$ defined by $m \phi$.

Definition 6.1. A subset $A \subset \mathcal{H}$ satisfies partial $C^{0}$-estimates at level $m$ if there exists $C>0$ such that $\left|P_{m}(\phi)-\phi\right| \leq C$ for all $\phi \in A$.

Now assume $X$ is Fano, and set $L:=-K_{X}$. Given a Kähler form $\alpha \in c_{1}(X)$, consider Aubin's continuity method

$$
\operatorname{Ric}\left(\omega_{t}\right)=t \omega_{t}+(1-t) \alpha
$$

It is well-known that there exists a unique maximal solution $\left(\omega_{t}\right)_{t \in[0, T)}$, where $0<T \leq 1$. The following important result, due to Székelyhidi [Szé16], confirms a conjecture of Tian.

Theorem 6.2. The set $A:=\left\{\omega_{t} \mid t \in[0, T)\right\}$ satisfies partial $C^{0}$-estimates at level $m$, for arbitrarily large positive integers $m$.

Given this result, we shall prove

Theorem 6.3. Any uniformly K-stable Fano manifold admits a Kähler-Einstein metric.

By working (much) harder, Datar and Székelyhidi [DSz15] have in fact been able to deduce from Theorem 6.2 a much better result dealing with K-polystability and allowing a compact group action. 
6.2. CM-stability and partial $C^{0}$-estimates. We first present in some detail well-known ideas due to Tian [Tia12, $\S 4.3]$. In this section, $(X, L)$ is an arbitrary polarized manifold.

Proposition 6.4. Assume that $(X, m L)$ is $C M$-stable, and that $A \subset \mathcal{H}$ satisfies partial $C^{0}$-estimates at level $m$. Then there exist $\delta, C>0$ such that $M \geq \delta J-C$ on $A$.

The proof, which is similar to the arguments in [Szé16, §5]. is based on two lemmas.

Lemma 6.5. For any two metrics $\phi, \psi \in \mathcal{H}$, we have

(i) $|J(\phi)-J(\psi)| \leq 2 \sup (\phi-\psi)$;

(ii) $M(\phi) \geq M(\psi)-C \sup |\phi-\psi|$ for some $C>0$ only depending on a one-sided bound (either upper or lower) for the Ricci curvature of the Kähler metric $d d^{c} \psi$.

Proof. Recall that

$$
E(\phi)-E(\psi)=\frac{1}{n+1} \sum_{j=0}^{n} V^{-1} \int_{X}(\phi-\psi)\left(d d^{c} \phi\right)^{j} \wedge\left(d d^{c} \psi\right)^{n-j}
$$

As a consequence, $|E(\phi)-E(\psi)| \leq \sup |\phi-\psi|$, and (i) follows immediately.

For (ii), we basically argue as in the proof of [Tia17, Lemma 3.1]. By the Chen-Tian formula 1.11, we have

$$
M(\phi)-M(\psi)=H_{\psi}(\phi)+\bar{S}(E(\phi)-E(\psi))+E_{\operatorname{Ric}\left(d d^{c} \psi\right)}(\psi)-E_{\operatorname{Ric}\left(d d^{c} \psi\right)}(\phi) .
$$

Here the entropy term $H_{\psi}(\phi)$ is non-negative, and we have

$$
E_{\operatorname{Ric}\left(d d^{c} \psi\right)}(\phi)-E_{\operatorname{Ric}\left(d d^{c} \psi\right)}(\psi)=\sum_{j=0}^{n-1} V^{-1} \int_{X}(\phi-\psi)\left(d d^{c} \phi\right)^{j} \wedge\left(d d^{c} \psi\right)^{n-j-1} \wedge \operatorname{Ric}\left(d d^{c} \psi\right) .
$$

Assume $\operatorname{Ric}\left(d d^{c} \psi\right) \leq C d d^{c} \psi$ for some constant $C>0$. We may then write

$$
\begin{aligned}
\left(d d^{c} \phi\right)^{j} \wedge\left(d d^{c} \psi\right)^{n-j-1} & \wedge \operatorname{Ric}\left(d d^{c} \psi\right) \\
\quad=C\left(d d^{c} \phi\right)^{j} & \wedge\left(d d^{c} \psi\right)^{n-j}-\left(d d^{c} \phi\right)^{j} \wedge\left(d d^{c} \psi\right)^{n-j-1} \wedge\left(C^{\prime} d d^{c} \psi-\operatorname{Ric}\left(d d^{c} \psi\right)\right),
\end{aligned}
$$

a difference of two positive measures of mass $C V$ and $C V+\left(L^{n-1} \cdot K_{X}\right)$, respectively, and the desired estimate follows.

The case where $\operatorname{Ric}\left(d d^{c} \psi\right) \geq-C^{\prime} d d^{c} \psi$ is treated similarly (and will anyway not be used in what follows).

We next recall a well-known upper bound for the Ricci curvature of restrictions of FubiniStudy metrics.

Lemma 6.6. We have $\operatorname{Ric}\left(d d^{c} \phi\right) \leq N_{m} d d^{c} \phi$ for all $\phi \in \mathcal{H}_{m}$.

Proof. Choose a basis of $H^{0}(X, m L)$, and let $\omega$ be the corresponding Fubini-Study metric on $\mathbb{P}:=\mathbb{P} H^{0}(X, m L)^{*}$. Its curvature tensor

$$
\Theta\left(T_{\mathbb{P}}, \omega\right) \in C^{\infty}\left(\mathbb{P}, \Lambda^{1,1} T_{\mathbb{P}}^{*} \otimes \operatorname{End}\left(T_{\mathbb{P}}\right)\right)
$$

is Griffiths positive and satisfies

$$
\operatorname{Tr}_{T_{\mathbb{P}}} \Theta\left(T_{\mathbb{P}}, \omega\right)=\operatorname{Ric}(\omega)=N_{m} \omega .
$$

For each complex submanifold $Y \subset \mathbb{P}$, the curvature of its tangent bundle $T_{Y}$ with respect to $\left.\omega\right|_{Y}$ satisfies $\Theta\left(T_{Y},\left.\omega\right|_{Y}\right) \leq\left.\Theta\left(T_{\mathbb{P}}, \omega\right)\right|_{T_{Y}}$ as $(1,1)$-forms on $Y$ with values in the 
endomorphisms of $T_{Y}$, as a consequence of a well-known curvature monotonicity property going back to Griffiths. We thus have

$$
\operatorname{Ric}\left(\left.\omega\right|_{Y}\right)=\operatorname{Tr}_{T_{Y}} \Theta\left(T_{Y},\left.\omega\right|_{Y}\right) \leq\left.\operatorname{Tr}_{T_{Y}} \Theta\left(T_{\mathbb{P}}, \omega\right)\right|_{T_{Y}}
$$

Using now $\Theta\left(T_{\mathbb{P}}, \omega\right) \geq 0$, we have on the other hand

$$
\left.\operatorname{Tr}_{T_{Y}} \Theta\left(T_{\mathbb{P}}, \omega\right)\right|_{T_{Y}} \leq \operatorname{Tr}_{T_{\mathbb{P}}} \Theta\left(T_{\mathbb{P}}, \omega\right)\left|Y=N_{m} \omega\right|_{Y}
$$

and hence

$$
\operatorname{Ric}\left(\left.\omega\right|_{Y}\right) \leq\left. N_{m} \omega\right|_{Y}
$$

Applying this to the images of $X \subset \mathbb{P}$ under projective transformations yields the desired result.

Proof of Proposition 6.4. Since $(X, m L)$ is CM-stable, there exist $\delta, C>0$ such that

$$
M\left(P_{m}(\phi)\right) \geq \delta J\left(P_{m}(\phi)\right)-C
$$

for all $\phi \in \mathcal{H}$. By assumption on $A$, we also have $\left|P_{m}(\phi)-\phi\right| \leq C$ for all $\phi \in A$, and by Lemma 6.6, the Ricci curvature of $d d^{c} P_{m}(\phi)$ is uniformly bounded above. Hence Lemma 6.5 shows, as desired, that there exists $C^{\prime}>0$ with $M(\phi) \geq \delta J(\phi)-C^{\prime}$ for all $\phi \in A$.

6.3. Proof of Theorem 6.3. Assume now that $X$ is a Fano manifold and set $L:=-K_{X}$. Consider the continuity method (6.1). Pick metrics $\psi$ and $\phi_{t}$ on $-K_{X}$ such that $\alpha=d d^{c} \psi$ and $\omega_{t}=d d^{c} \phi_{t}$, respectively. After adding a constant to $\phi_{t}$, 6.1 may be written

$$
\left(d d^{c} \phi_{t}\right)^{n}=e^{-2\left(t \phi_{t}+(1-t) \psi\right)} .
$$

We recall the proof of the following well-known monotonicity property.

Lemma 6.7. The function $t \rightarrow M\left(\phi_{t}\right)$ is non-increasing.

Proof. We have

$$
\begin{aligned}
-\frac{d}{d t} M\left(\phi_{t}\right) & =n V^{-1} \int_{X} \dot{\phi}_{t}\left(\operatorname{Ric}\left(\omega_{t}\right) \wedge \omega_{t}^{n-1}-\omega_{t}^{n}\right) \\
& =n V^{-1}(1-t) \int_{X} \dot{\phi}_{t} d d^{c}\left(\psi-\phi_{t}\right) \wedge\left(d d^{c} \phi_{t}\right)^{n-1} \\
& =n V^{-1}(1-t) \int_{X}\left(\psi-\phi_{t}\right) d d^{c} \dot{\phi}_{t} \wedge\left(d d^{c} \phi_{t}\right)^{n-1}
\end{aligned}
$$

Since $d^{c}$ is normalized so that $d d^{c}=\frac{i}{\pi} \partial \bar{\partial}$, we have

$$
n \frac{d d^{c} \dot{\phi}_{t} \wedge \omega_{t}^{n-1}}{\omega_{t}^{n}}=\operatorname{tr}_{\omega_{t}} d d^{c} \dot{\phi}_{t}=-\frac{1}{2 \pi} \Delta_{t}^{\prime \prime} \dot{\phi}_{t}
$$

with $\Delta_{t}^{\prime \prime}$ denoting the $\bar{\partial}$-Laplacian with respect to $\omega_{t}$. On the other hand, differentiating 6.3 yields

$$
n d d^{c} \dot{\phi}_{t} \wedge \omega_{t}^{n-1}=2\left(\psi-\phi_{t}-t \dot{\phi}_{t}\right) \omega_{t}^{n}
$$

and hence

$$
\psi-\phi_{t}=\left(t-\frac{1}{\pi} \Delta_{t}^{\prime \prime}\right) \dot{\phi}_{t}
$$


We get

$$
\begin{aligned}
-\frac{d}{d t} M\left(\phi_{t}\right) & =\frac{1-t}{2 \pi} \int_{X}\left(\left(\frac{1}{\pi} \Delta_{t}^{\prime \prime}-t\right) \dot{\phi}_{t}\right)\left(\Delta_{t}^{\prime \prime} \dot{\phi}_{t}\right) \operatorname{MA}\left(\phi_{t}\right) \\
& =\frac{1-t}{2 \pi} \int_{X}\left\langle\left(\frac{1}{\pi} \Delta_{t}^{\prime \prime}-t\right) \bar{\partial} \dot{\phi}_{t}, \bar{\partial} \dot{\phi}_{t}\right\rangle_{\omega_{t}} \operatorname{MA}\left(\phi_{t}\right) .
\end{aligned}
$$

Since $\operatorname{Ric}\left(\omega_{t}\right) \geq t \omega_{t}$, the $\bar{\partial}$-Laplacian $\Delta_{t}^{\prime \prime}$ satisfies $\frac{1}{\pi} \Delta_{t}^{\prime \prime} \geq t$ on $(0,1)$-forms, and the last integral is thus nonnegative. Indeed, this follows from the Bochner-Kodaira-Nakano identity applied to

$$
C^{\infty}\left(X, \Lambda^{0,1} T_{X}^{*}\right) \simeq C^{\infty}\left(X, \Lambda^{n, 1} T_{X}^{*} \otimes K_{X}^{*}\right)
$$

with the fiber metric $\psi_{t}=-\frac{1}{2} \log \omega_{t}^{n}$ on $K_{X}^{*}=-K_{X}$, with curvature $d d^{c} \psi_{t}=\operatorname{Ric}\left(\omega_{t}\right)$.

We may now complete the proof of Theorem 6.3. By Corollary E, $\left(X,-m K_{X}\right)$ is CMstable for all $m$ divisible enough. Theorem 6.2 and Proposition 6.4 therefore yield $\delta, C>0$ such that $M\left(\phi_{t}\right) \geq \delta J\left(\phi_{t}\right)-C$ along Aubin's continuity method. Since $M\left(\phi_{t}\right)$ is bounded above by Lemma 6.7, it follows that $J\left(\phi_{t}\right)$ remains bounded. By [Tia00, Lemma 6.19], the oscillation of $\phi_{t}$ is bounded, and well-known arguments allow us to conclude, see [Tia00, $\S 6.2]$.

\section{REFERENCES}

[Ant] S. Antonakoudis, Valuative criteria of separatedness and properness. https://www.dpmms.cam.ac.uk/ sa443/papers/criteria.pdf

[Berk90] V. Berkovich. Spectral theory and analytic geometry over non-Archimedean fields. Mathematical Surveys and Monographs, vol. 33. American Mathematical Society, Providence, RI, 1990.

[Berk09] V. G. Berkovich. A non-Archimedean interpretation of the weight zero subspaces of limit mixed Hodge structures. In Algebra, arithmetic, and geometry: in honor of Yu. I. Manin. Progr. Math., vol 269, 49-67. Birkhäuser, Boston, MA, 2009.

[Berm16] R. J. Berman. K-polystability of $\mathbb{Q}$-Fano varieties admitting Kähler-Einstein metrics. Invent. Math. 203 (2016), 973-1025.

[BB14] R. J. Berman and B. Berndtsson. Convexity of the K-energy on the space of Kähler metrics. arXiv: 1405.0401.

[BBEGZ11] R. J. Berman, S. Boucksom, P. Eyssidieux, V. Guedj and A. Zeriahi. Kähler-Einstein metrics and the Kähler-Ricci flow on log Fano varieties. arXiv:1111.7158.

[BBGZ13] R. J. Berman, S. Boucksom, V. Guedj and A. Zeriahi. A variational approach to complex MongeAmpère equations. Publ. Math. Inst. Hautes Études Sci. 117 (2013), 179-245.

[BBJ15] R. J. Berman, S. Boucksom and M. Jonsson. A variational approach to the Yau-Tian-Donaldson conjecture. arXiv: 1509.04561.

[BDL16] R. J. Berman, T. Darvas and C. H. Lu. Regularity of weak minimizers of the K-energy and applications to properness and K-stability. arXiv:1602.03114.

[BFJ16] S. Boucksom, C. Favre and M. Jonsson. Singular semipositive metrics in non-Archimedean geometry. J. Algebraic Geom. 25 (2016), 77-139.

[BFJ15a] S. Boucksom, C. Favre and M. Jonsson. Solution to a non-Archimedean Monge-Ampère equation. J. Amer. Math. Soc., 28 (2015), 617-667.

[BHJ15] S. Boucksom, T. Hisamoto and M. Jonsson. Uniform K-stability, Duistermaat-Heckman measures and singularities of pairs. arXiv: 1504.06568. To appear in Ann. Inst. Fourier.

[BJ16a] S. Boucksom and M. Jonsson. Tropical and non-Archimedean limits of degenerating families of volume forms. arXiv:1605.05277. To appear in J. Éc. polytech. Math.

[BJ16b] S. Boucksom and M. Jonsson. Singular semipositive metrics on line bundles on varieties over trivially valued fields. In preparation. 
[Che00] X.X. Chen. On the lower bound of the Mabuchi energy and its application. Int. Math. Res. Not. (2000), no. 12, 607-623.

[CDS15] X.X. Chen, S. K. Donaldson and S. Sun. Kähler-Einstein metrics on Fano manifolds, I-III. J. Amer. Math. Soc. 28 (2015), 183-197, 199-234, 235-278.

[CSW15] X.X. Chen, S. Sun and B. Wang. Kähler-Ricci flow, Kähler-Einstein metric, and K-stability. arXiv: 1508.04397.

[DR15] T. Darvas and Y. A. Rubinstein Tian's properness conjectures and Finsler geometry of the space of Kähler metrics. arXiv:1506.07129. To appear in J. Amer. Math. Soc.

[DSz15] V. Datar and G. Székelyhidi. Kähler-Einstein metrics along the smooth continuity method. Geom. Funct. Anal. 26 (2016), 975-1010.

[Der15] R. Dervan. Uniform stability of twisted constant scalar curvature Kähler metrics. Int. Math. Res. Not. (2016), no. 15, 4728-4783.

[DR16] R. Dervan and J. Ross. K-stability for Kähler manifolds. arXiv:1602.08983.

[Din88] W.-Y. Ding. Remarks on the existence problem for positive Kähler-Einstein metrics. Math. Ann. 282 (1988) 463-471.

[DT92] W.-Y. Ding and G. Tian. Kähler-Einstein metrics and the generalized Futaki invariant. Invent. Math. 110 (1992), 315-335.

[Don99] S. K. Donaldson. Symmetric spaces, Kähler geometry and Hamiltonian dynamics. Northern California Symplectic Geometry Seminar, 13-33. Amer. Math. Soc. Transl. Ser. 2, 196. Amer. Math. Soc., Providence, RI, 1999.

[Don02] S. K. Donaldson. Scalar curvature and stability of toric varieties. J. Differential Geom. 62 (2002), 289-349.

[Don05] S. K. Donaldson. Lower bounds on the Calabi functional. J. Differential Geom. 70 (2005), 453472 .

[Don12] S.K. Donaldson. Stability, birational transformations and the Kahler-Einstein problem. Surv. Diff. Geom., vol. 17, International Press, Boston, MA (2012), 203-228.

[Elk89] R. Elkik. Fibrés d'intersections et intégrales de classes de Chern. Ann. Sci. École Norm. Sup. (4) 22 (1989), 195-226.

[Elk90] R. Elkik. Métriques sur les fibrés d'intersection. Duke Math. J. 61 (1990), 303-328.

[Fuj15] K. Fujita. Optimal bounds for the volumes of Kähler-Einstein Fano manifolds. arXiv: 1508.04578.

[Fuj16] K. Fujita. A valuative criterion for uniform K-stability of $\mathbb{Q}$-Fano varities. arXiv:1602.00901v1.

[His16] T. Hisamoto. On the limit of spectral measures associated to a test configuration of a polarized Kähler manifold. J. Reine Angew. Math. 713 (2016), 129-148.

[Jon16] M. Jonsson. Degenerations of amoebae and Berkovich spaces. Math. Ann. 364 (2016), $293-311$.

[Kap13] H.M. Kapadia. Deligne pairings and discriminants of algebraic varieties. arXiv:1312.7870.

[Kem] G. Kempf. Algebraic varieties. LMS Lecture Note Series 172. Cambridge University Press, Cambridge, 1993.

[Li12] C. Li. Kähler-Einstein metrics and K-stability. Ph.D. Thesis, Princeton University, 2012.

[LX14] C. Li and C. Xu. Special test configurations and K-stability of Fano varieties. Ann. of Math. 180 (2014), 197-232.

[Li14] L. Li. Subharmonicity of conic Mabuchi's functional, I. arXiv:1511.00178.

[Mab87] T. Mabuchi. Some symplectic geometry on compact Kähler manifolds. I. Osaka J. Math. 24 (1987), 227-252.

[Mor99] A. Moriwaki. The continuity of Deligne's pairing. Internat. Math. Res. Notices 19 (1999) 10571066.

[MFF $] \quad$ D. Mumford, J. Fogarty and F. Kirwan. Geometric invariant theory. Third edition. Ergebnisse der Mathematik und ihrer Grenzgebiete (2), 34. Springer-Verlag, Berlin, 1994.

[MG00] E. Muñoz Garcia. Fibrés d'intersection. Compositio Math. 124 (2000) 219-252.

[Oda13] Y. Odaka. A generalization of the Ross-Thomas slope theory. Osaka J. Math. 50 (2013), 171-185.

[Pau04] S. T. Paul. Geometric analysis of Chow Mumford stability. Adv. Math. 182 (2004), 333-356.

[Pau12] S. T. Paul. Hyperdiscriminant polytopes, Chow polytopes and Mabuchi energy asymptotics. Ann. of Math. (2) $\mathbf{1 7 5}$ (2012), 255-296.

[Pau13] S. T. Paul. Stable pairs and coercive estimates for the Mabuchi functional. arXiv:1308.4377. 
[PT06] S. T. Paul and G. Tian. CM Stability and the Generalized Futaki Invariant I. arXiv:math/0605278.

[PT09] S. T. Paul and G. Tian. CM Stability and the Generalized Futaki Invariant II. Astérisque No. 328 (2009), 339-354.

[PRS08] D. H. Phong, J. Ross, and J. Sturm. Deligne pairings and the Knudsen-Mumford expansion. J. Differential Geom. 78 (2008), 475-496.

[PSSW08] D. H. Phong, J. Song, J. Sturm and B. Weinkove. The Moser-Trudinger inequality on KählerEinstein manifolds. Amer. J. Math. 130 (2008), 1067-1085.

[PS04] D. H. Phong and J. Sturm. Scalar curvature, moment maps, and the Deligne pairing. Amer. J. Math. 126 (2004), no. 3, 693-712.

[Sem92] S. Semmes. Complex Monge-Ampère and symplectic manifolds. Amer. J. Math. 114 (1992), 495-550.

[SD16] Z. Sjöström Dyrefelt. K-semistability of cscK manifolds with transcendental cohomology class. arXiv: 1601.07659.

[Stol66] W. Stoll. The continuity of the fiber integral. Math. Z. 95 (1966), no.2, 87-138.

[Stop09] J. Stoppa. K-stability of constant scalar curvature Kähler manifolds. Adv. Math. 221 (2009) 1397-1408.

[Szé06] G. Székelyhidi. Extremal metrics and K-stability. Ph.D Thesis. arXiv:math/0611002.

[Szé15] G. Székelyhidi. Filtrations and test-configurations. With an appendix by S. Boucksom. Math. Ann. 362 (2015), 451-484.

[Szé16] G. Székelyhidi. The partial $C^{0}$-estimate along the continuity method. J. Amer. Math. Soc. 29 (2016), 537-560.

[Tho06] R. Thomas. Notes on GIT and symplectic reduction for bundles and varieties. Surveys in Differential Geometry, Vol. 10, 221-273. Int. Press, Somerville, MA, 2006.

[Tia97] G. Tian. Kähler-Einstein metrics with positive scalar curvature. Inv. Math. 130 (1997), 239-265.

[Tia00] G. Tian. Canonical metrics in Kähler geometry. Notes taken by Meike Akveld. Lectures in Mathematics ETH Zürich. Birkhäuser Verlag, Basel, 2000.

[Tia12] G. Tian. Existence of Einstein metrics on Fano manifolds. Metric and differential geometry, 119-159, Progr. Math., 297, Birkhäuser/Springer, Basel, 2012.

[Tia17] G. Tian. K-stability implies CM-stability. In: Bost J.-B., Hofer H., Labourie F., Le Jan Y., Ma X., Zhang W. (eds) Geometry, Analysis and Probability. Progress in Mathematics 310. Birkhäuser, Cham.

[Tia15] G. Tian. K-stability and Kähler-Einstein metrics. Comm. Pure Appl. Math. 68 (2015), 10851156.

[Wan12] X.-Wang. Height and GIT weight. Math. Res. Lett. 19 (2012), 909-926.

[Zha96] S.-W. Zhang. Heights and reductions of semi-stable varieties. Compositio Math. 104 (1996), $77-105$.

CNRS-CMls, École Polytechnique, F-91128 Palaiseau Cedex, France

E-mail address: sebastien.boucksom@polytechnique.edu

Graduate School of Mathematics, Nagoya University, Furocho, Chikusa, Nagoya, Japan

E-mail address: hisamoto@math.nagoya-u.ac.jp

Dept of Mathematics, University of Michigan, Ann Arbor, Mi 48109-1043, USA

Mathematical Sciences, Chalmers University of Technology and University of Gothenburg, SE-412 96 GÖTEBorg, SwEDEN

E-mail address: mattiasj@umich.edu 